\title{
Modulatory Effect of Peripheral Magnetic and Neuromuscular Electrical Stimulation on Cortical Excitability: A Functional Near-Infrared Spectroscopy Study
}

\section{Fengyun Yu}

Huashan Hospital Fudan University Department of Rehabilitation https://orcid.org/0000-0001-5559-

5775

\section{Weining Wang}

Fudan University Huashan Hospital Department of rehabilitation medicine

\section{Sijie Liang}

Fudan University Huashan Hospital Department of Rehabilitation medicine

\section{Ce Li}

Fudan University Huashan Hospital Department of Rehabilitation medicine

Shan Tian

Fudan University Huashan Hospital Department of Rehabilitation medicine

\section{Ruiping Hu}

Fudan University Huashan Hospital Department of Rehabilitation medicine

\section{Yi Wu}

Fudan University Huashan Hospital Department of Rehabilitation medicine

\section{Yulian Zhu ( $\square$ zyljully@163.com )}

Fudan University Huashan Hospital Department of Rehabilitation medicine

\section{Research}

Keywords: neuromuscular electrical stimulation, peripheral magnetic stimulation, corticomotor excitability, functional near-infrared spectroscopy, cortical activity.

Posted Date: May 13th, 2021

DOl: https://doi.org/10.21203/rs.3.rs-504685/v1

License: (9) This work is licensed under a Creative Commons Attribution 4.0 International License. Read Full License 


\section{Modulatory effect of peripheral magnetic and neuromuscular}

2 electrical stimulation on cortical excitability: a functional

3 near-infrared spectroscopy study

4 Fengyun $\mathrm{Yu}^{1}$,\#, Weining Wang, \#, Sijie Liang ${ }^{1}, \mathrm{Ce} \mathrm{Li}^{1}$, Shan Tian ${ }^{1}$, Ruiping Hu${ }^{1}$, Yi

$5 \mathrm{Wu}^{1}$, Yulian $\mathrm{Zhu}^{1, *}$

6

$7{ }^{1}$ Department of Rehabilitation medicine, Huashan Hospital, Fudan University,

8 Shanghai, China.

10 *Corresponding author:

11 Yulian Zhu

12 Department of Rehabilitation Medicine, Huashan Hospital, Fudan University

13 12\# Wulumuqi middle road, Jing'an, shanghai, China

14 E-mail: zyljully@163.com

15

$16{ }^{\#}$ These authors participated equally in this study 
ABSTRACT

Background: The present study was designed to investigate the effects of neuromuscular electrical stimulation (NMES) and peripheral magnetic stimulation (PMS) applied to the wrist extensor muscle on the cortical activity of healthy adults by using fNIRS.

Methods: Fifteen healthy adult subjects ( 7 males, mean age: $27.13 \pm 4.52$ years) all received two different conditions of peripheral muscle stimulation in random order: (1) NMES and (2) PMS. The sessions were separated by at least $48 \mathrm{~h}$ as a washout period. During muscle stimulation, the motor evoked potential (MEP) of the left primary motor cortex (M1) was measured by transcranial magnetic stimulation (TMS) and the concentration of oxygenated $(\mathrm{HbO})$ and deoxygenated $(\mathrm{HbR})$ hemoglobin detected by fNIRS were used to evaluate the excitability and the activity of the cortex.

Results: After the stimulation of the wrist extensor, the MEP amplitude in the left M1 area did not change in both conditions, and there was no difference between NMES and PMS condition. NMES reduced $\mathrm{HbO}$ values of several channels in the Prefrontal cortex (PFC), Somatosensory motor cortex (SMC) and Occipital cortex (OC), and HbR valus of several channels in the PFC and SMC. During the PMS stimulation period, the $\mathrm{HbO}$ value of all brain areas did not change significantly, while the HbR value of the SMC area decreased. The $\mathrm{HbO}$ and $\mathrm{HbR}$ value of the channels in the SMC did not differ between NMES and PMS. Inter-region of interest and inter-channel analysis between NMES and PMS showed no difference in functional connectivity.

Conclusions: In the case of wrist extensor muscle stimulation, both NMES and PMS can induce cortical activation. PMS targeted to increases the activity of the contralateral SMC, while NMES increased contralateral SMC activity and negatively activated the $\mathrm{PFC}$ and $\mathrm{OC}$.

Keywords: neuromuscular electrical stimulation; peripheral magnetic stimulation; corticomotor excitability; functional near-infrared spectroscopy; cortical activity. 


\section{Introduction}

Neuromuscular electrical stimulation (NMES) is a classic non-invasive peripheral stimulation (NIPS) method. It is performed by applying an electric current to the muscle or peripheral nerve. In general, NMES has been applied alone or in combination with other rehabilitation measures for rehabilitation after stroke[1, 2], chronic obstructive pulmonary disease[3], muscle weakness, and musculoskeletal diseases (low back pain, hip and knee arthroplasty, anterior cruciate ligament)[4]. In essence, the mechanism of NMES is that electrical current delivery to neuromuscular tissue causes the depolarization of the motor axons to indirectly activate fiber contraction, When the intensity of NMES exceeds the motor threshold (MT), an upward afferent signal is generated, and then the muscle contraction induced by the electrical stimulation causes a re-afferent. Transcranial magnetic stimulation (TMS) [5] and Electroencephalography (EEG) studies[6] have found that NMES can affect the excitability of the primary sensory (S1) and motor cortex (M1) when applied to the first dorsal interosseous (FDI) or abductor pollicis brevis (APB) muscle. This excitatory change is generally believed to reflect the restoration of brain function and reorganization of brain networks[7]. Previous studies demonstrated that peripheral stimulation may eventually affect cerebral functional recovery and reconfiguration of brain networks $[8,9]$, thereby improving motor performance in patients with brain injury[10].

Peripheral magnetic stimulation (PMS) is a new NIPS technique that applies highintensity magnetic field to the periphery. The application of its magnetic coil to the spinal root, nerve, or muscle belly has a similar effect to NMES[11]. Moreover, PMS does not require skin contact and does not cause pain during the procedure, which makes it applicable to patients with paresthesia and to perform deep stimulation. These unique advantages of PMS make it an alternative to NMES. Moreover, PMS can cause changes in cortical excitability by inducing proprioceptive input to the central nervous system (CNS) through magnetic stimulation. It has two different mechanisms: 1) the rhythmic contraction and relaxation of muscles induced by indirect stimulation lead to adequate activation of mechanoreceptors (fiber groups: Ia, Ib, II), and 2) direct stimulus of sensory motor fibers induce inadequate activation of sensorimotor nerve fibers [12]. Considering the after-effect and no pain in clinical application, PMS is a new rehabilitation technology with more potential than NMES[13, 14].

Functional near-infrared spectroscopy (fNIRS) is a non-invasive, real-time, and continuous optical technique that is used to measure cortical activities by measuring oxygenated ([HbO]) and deoxygenated ([HbR]) hemoglobin concentrations during task. That is, neural activity rapidly increases local blood flow to meet transient changes in local brain energy requirements[15]. As a new detection method, fNIRS has higher temporal resolution and higher tolerance to motion artifacts than fMRI, but very low 
temporal resolution compared with EEG. Subjects can be tested in a more comfortable position compared with the conventional testing technique of fMRI[16, 17].

NMES and PMS can both alter cortical excitability and promote neuroplasticity, but the mechanism remains unknown. PMS has been used to improve function in the paretic upper and lower limbs after stroke[18]. Given the unique advantages of PMS, we believe that PMS may be a better treatment method compared with NMES. However, quantitative analyses and comparative studies of the effect of NMES and PMS on cortical excitability are lacking, and their mechanisms on cortical excitability are not clear. To determine whether PMS has the potential to replace NMES in peripheral treatment, this study aimed to compare the effects of NMES and PMS on cortical excitability and cortical activation when applied to the dominant wrist extensor muscles.

\section{Materials and methods}

\section{Participants}

Fifteen healthy right-handed volunteers ( 7 males, mean aged: $27.13 \pm 4.52$ years) participated in this experiment. None of the subjects had any health problems, such as neurological diseases, mental illness, upper limb sensory disorder, movement disorder, or any contraindications to TMS. Before the experiment, we explained this purpose of the experiment and the sensation during the stimulation to the participants. This study was approved by the Huashan Hospital Institutional Review Board, Fudan University, and written informed consent was obtained from all subjects.

\section{Experiment design}

Our study is an exploratory crossover design. To exclude interference from the external environment, the experiment was conducted in a separate, quiet, and darkroom. Each subject received two different types of muscle stimulation: NMES and PMS with randomized order. To avoid cross-over effects, the two conditions were spaced at least $48 \mathrm{~h}$ apart. Before the first session, all subjects were required to fill in personal information, including name, age, height, weight, dominant hand, and health status. After each stimulation condition, subjects performed a self-assessment questionnaire to evaluate the comfort of the two stimulation conditions. The content of the questionnaire included headache, skin irritation, noise, negative mood swings, muscle twitching, drowsiness, numbness, and heart rate during muscle stimulation. Motor evoked potential (MEP) was used to assess the cortical excitability before (pre) and after (post) each session. The fNIRS signal was measured before stimulation (resting-state fNIRS) and during stimulation task for both conditions (Figure 1). In this study, resting-state 
fNIRS (5 min) was used to detect whether the functional connectivity strengths of subjects were consistent before receiving different muscle stimulation, to avoid individual differences caused by time. Throughout the experiment, subjects were kept relaxed with their eyes open in armchair.

\section{Peripheral muscle intervention}

130 NMES was provided using ES-521 Electrotherapy (ITO Co., Ltd., Tokyo, Japan) with

131 two independent channels. Only one channel was used in the experiment. Stimulating 132 electrodes were placed distal to the common extensor origin and halfway down the extensor surface of the right hand's forearm (both cover the extensor carpi ulnar and extensor carpi radialis). The protocol was conducted in a frequency of $50 \mathrm{~Hz}$ with on: off stimulation time of $1: 3$ (10 s of stimulation and $30 \mathrm{~s}$ of rest) cycle for $10 \mathrm{~min}$ (repeated 15 times), ramp-up and ramp-down $1 \mathrm{~s}$. The intensity of the electrical current was adjusted to induce wrist extension to reach the maximum motion as far as possible while the subject remained comfortable without feeling pain (mean $12.30 \pm 3.78 \mathrm{~mA}$ ).

The PMS coil center was applied to the point where the forearm muscle contraction was most obvious when the subject active wrist extension at the $120^{\circ}$ elbow flexion and forearm pronation position, using an OSF-pTMS magnetic stimulator (O.SELF Company, Wuhan, China) with a figure of eight shaped coil. The PMS protocol was applied at a frequency of $10 \mathrm{~Hz}$ with 15 repetitions of $10 \mathrm{~s}$ on/30 s off. A total of 1500 pulses were applied, which lasted for $10 \mathrm{~min}$. The intensity of magnetic stimulation was adjusted (mean $30.47 \% \pm 4.78 \% \mathrm{MSO}$ ) to induce maximum wrist extension without causing discomfort to the subjects.

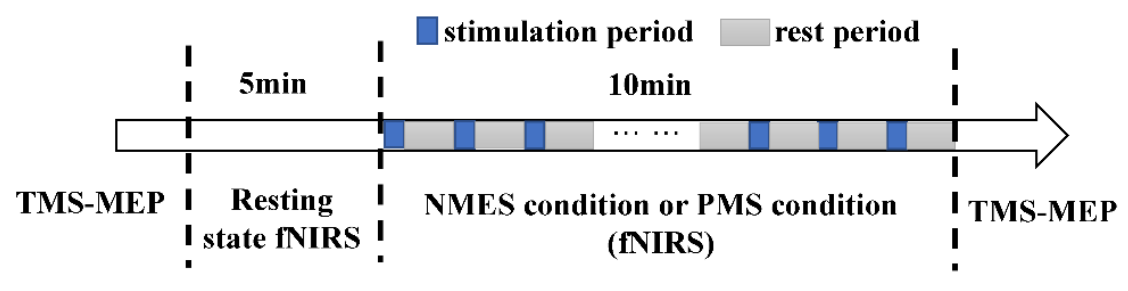

Figure 1. TMS-MEP and resting state fNIRS were assessed at the beginning of each condition, then one of the two muscle stimulation interventions (i.e. NMES or PMS) was applied, and fNIRS was also assessed during stimulation. After the intervention, cortical excitability was reassessed. 


\section{Measurement of motor evoked potentials}

155 Transcranial magnetic stimulation (TMS) was performed with an OSF-pTMS magnetic

156 stimulator (O.SELF Company, Wuhan, China) with a figure-of-eight-shaped coil, 157 which can be used in the single-pulse assessment paradigm and rTMS paradigm. To 158 assess cortical excitability, a pair of $\mathrm{Ag} / \mathrm{AgCl}$ surface electrodes were placed on the

159 belly of the FDI muscle of the right hand, and the surface electromyography signals can 160 be observed on a computer screen. The coil was positioned at a $45^{\circ}$ tangent to the skull 161 in the left M1, and the center of the coil was moved within a range of $0.5 \mathrm{~cm}$ each time 162 in the motor cortex until we found the optimal site that could induce the maximum MEP 163 amplitude. The resting motor threshold (RMT) and motor evoked potentials (MEPs) 164 were examined by single-pulse TMS parameters. The RMT was defined as the minimal 165 stimulation intensity that can induce at least five trials with MEP peak-peak wave 166 amplitude $>50 \mu \mathrm{V}$ when the FDI muscles were continuously stimulated for 10 trials. 167 In both conditions, MEPs amplitude was recorded before resting-state fNIRS 168 monitoring and immediately after muscle stimulation. The MEP measured intensity 169 was the intensity with peak-peak wave value at $1 \mathrm{mV}$ intensity before the intervention. 170 Ten consecutive TMS pulses were spaced by at least $5 \mathrm{~s}$.

\section{2 fNIRS equipment}

173 A continuous-wave (CW), 64-multichannel fNIRS system (NirSmart, Danyang 174 Huichuang Medical Equipment, China) was utilized to measure [HbO] and [HbR] at 175 the resting state and during muscle stimulation with two wavelengths of 730 and 850 $176 \mathrm{~nm}$, and the sample rate was $11 \mathrm{~Hz}$. A total of 24 light sources and 24 light detectors 177 were symmetrically positioned over the whole brain regions, forming a total of 64 178 channels. Each channel was composed of the light source probe and the detector probe 179 with a fixed distance of $3 \mathrm{~cm}$, which can detect cortical activity with a depth of about $180 \quad 1.5-2 \mathrm{~cm}$ (Figure 2). 


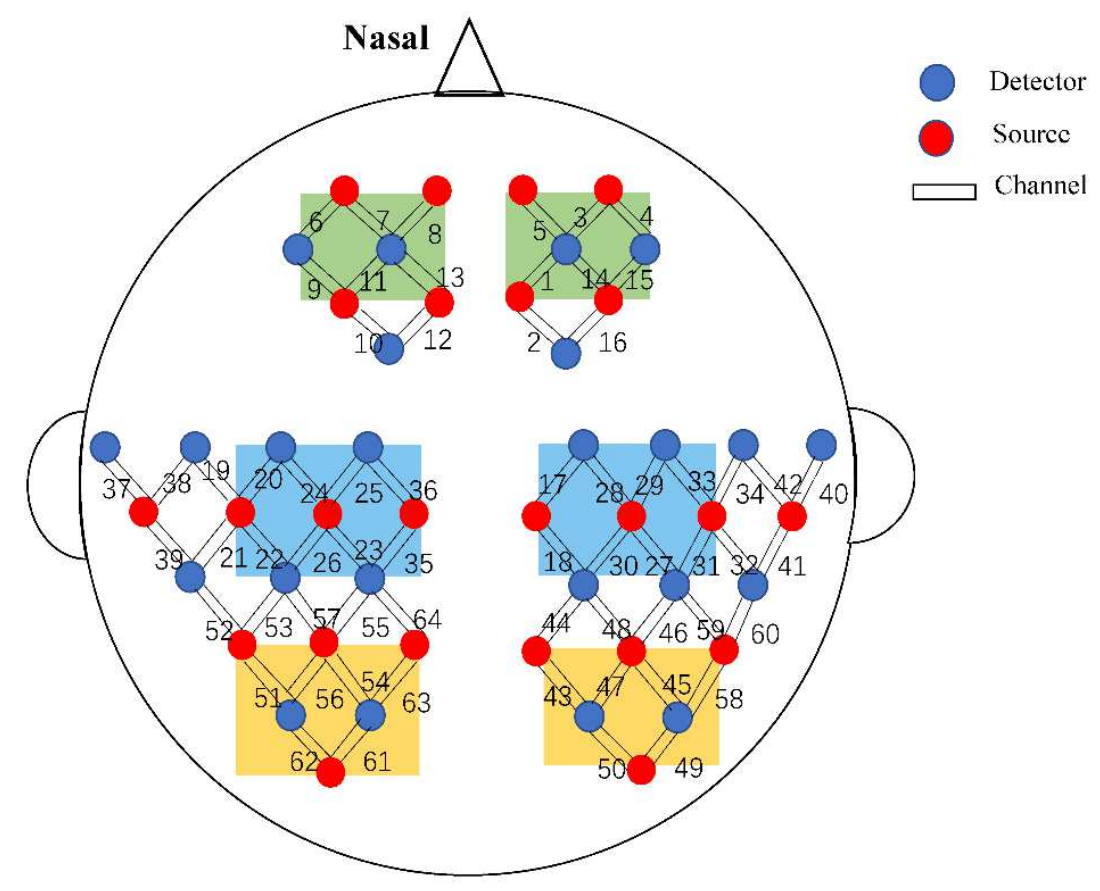

183 Figure 2. Probes placement. The locations of fNIRS detectors and sources are indicated

184 by the blue and red circles, respectively, and the numbers between the circles indicate the channel numbers. The distance between the luminous source and the detector is 3 $\mathrm{cm}$. According to the MNI spatial coordinates, the channels in the green region are located in the PFC, the channels in the blue region are located in the SMC, and the channels in the yellow region are located in the OC. Channels 23 and 35 correspond to the left forearm motor cortex, channels 18 and 30 correspond to the right forearm motor cortex. There are 64 channels in total, and only 40 channels in the color covered area are used for observation and analysis.

\section{fNIRS preprocessing}

194 Data analysis was mainly conducted using HomER (version 2.8), a MATLAB-based graphical user interface program that is principally designed for CW NIRS measurements [19]. The HomER program is freely available (http://www.nmr.mgh.harvard.edu/PMI/resources/homer/home.htm).

HomER provides the user a wide selection of function processing tools to choose from, depending on their needs. In this study, we first used the hmrIntensity2OD Utility

200 function to converts the raw optical intensity into OD optical density data. Then, the 201 hmrMotionArtifactByChannel tool was used to identify motion artifacts in the data matrix. STDev-thresh was set at 10, and AMP-thresh was set at 5. Motion artifacts were removed by using filtering methods based on spline interpolation. Bandpass filtering 
was used to remove unwanted specific frequency content. According to the muscle stimulation protocol frequency, we set the high pass filter at $0.01 \mathrm{~Hz}$ and the low pass filter at $0.1 \mathrm{~Hz}$. The hmrOD2Conc function was used to convert the signals into oxyhemoglobin and deoxyhemoglobin via the Beer-Lambert equation, partial pathlength factors for each wavelength was 6.0. Finally, the hmrBlockAvg function was used to average the time series data at -5 to $40 \mathrm{~s}$, the baseline of the average is set to 0 by subtracting the mean of the average for -5 to $0 \mathrm{~s}$.

211 NirSpark (NirSmart, Danyang Huichuang Medical Equipment, China), which also needs to run in MATLAB, was used to analyze brain functional connections. NirSpark provides General Linear Model (GLM) analysis and brain Network analysis. Data preprocessing was performed in the data preprocessing interface section of NirSpark with the same parameter values as applied in Homer2 (STDev-thresh was 10, AMPthresh was 5, hpf was $0.01 \mathrm{~Hz}$, lpf was $0.1 \mathrm{~Hz}$ and ppf was 6). GLM was applied to estimate cortical layers' $\mathrm{HbO}$ response during the stimulation tasks and the correlation between the time courses, including individual subject and condition levels, the beta value for the corresponding conditions was obtained. The full width at half maximum Gaussian smoothing with $4 \mathrm{~s}$ was used to correct the short-time high-frequency noise in the $\mathrm{HbO}$ signal. According to the MNI spatial coordinates of source-detector probes, target channels (40 channels of color coverage area) were selected and divided them into six regions of interest (ROIs): left prefrontal cortex (PFC) ch6, ch7, ch8, ch9, ch11, ch13), right PFC (ch1, ch3, ch4, ch5, ch14, ch15), left somatosensory motor cortex (SMC) (ch20, ch22, ch23, ch24, ch25, ch26, ch35, ch36), right SMC (ch17, ch18, ch27, ch28, ch29, ch30, ch31, ch33), left occipital cortex (OC) (ch51, ch54, ch56, ch61, ch62, ch63), and right OC (ch43, ch45, ch47, ch49, ch50, ch58). NirSpark's network analysis maps the connections of inter-ROIs and inter-channel (similarity threshold was set as $0.5,0.6,0.7$, and 0.8 , respectively) during different stimulation conditions. And the ROI-ROI connectivity and channel-channel connectivity between the NMES and PMS conditions were performed by t-test for both resting state fNIRS and stimulation fNIRS.

The SMC is an important brain functional region that integrates learning and motor tasks and responds quickly to peripheral and central operations. The changes in cortical activation of the SMC region during peripheral stimulation of wrist extensor muscle are important regions to observed in this study. According to the MNI spatial coordinates of channels, ch 23 and ch 25 are located in the left forearm motor cortex, ch18 and ch30 are located in the right forearm motor cortex. 


\section{Statistical analysis}

IBM SPSS 22 (Statistical Package for Social Sciences) was used for data analysis. Shapiro-Wilk test was used to validate the normality of all data. Data are presented as mean \pm standard deviation. Changes in cortical excitability were calculated by dividing the MEP amplitude after the intervention by MEP amplitude at baseline (MEP\% of baseline). The paired T-test was used to compare MEP amplitude before and after intervention and MEP\% of the baseline between two peripheral muscle stimulation conditions.

According to the stimulation task, the fNIRS values during stimulation were divided into stimulation period and rest period. The average amplitude of the $\mathrm{HbO}$ value and $\mathrm{HbR}$ value across the task period of 5 to $10 \mathrm{~s}$ was utilized as an index of cortical activity for the stimulation period. At the end of the stimulation cycle, 35 to $40 \mathrm{~s}$ away from the task period, cortical activity in this time period has ample time to fall back to the resting state, so the average amplitude of the $\mathrm{HbO}$ value and $\mathrm{HbR}$ value during the 35 to $40 \mathrm{~s}$ task period were used as the rest period. The $\mathrm{HbO}$ value and $\mathrm{HbR}$ value of the ROI were calculated based on the average value of all channels in the region. Paired t-test was performed on the $\mathrm{HbO}$ value and $\mathrm{HbR}$ value between the stimulation period and the rest period to compare the activation of channels in the PFC, SMC, and OC regions of the NMES and PMS conditions. Paired T-test was used to test the differences of the $\mathrm{HbO}$ and $\mathrm{HbR}$ value in SMCs' channels between the NMES and PMS stimulation periods. Pearson's correlation analysis was used to analyze the relationship between MEP amplitude changes and intervention intensity, the relationship between MEP amplitude changes and baseline RMT, and the relationships of MEP\% of the baseline between two stimulation conditions. The Benjamini-Hochberg method was used to correct for multiple comparisons. The significant differences of all tests were defined as p-value $<0.05$.

\section{Results}

Overall, Data from all 15 subjects aged $27.13 \pm 4.52$ years were included in the experiment. Their body mass was $62.14 \pm 17.47 \mathrm{~kg}$, the body mass index value was $22.11 \pm 4.18$, and the average education years was 16.67 years. The characteristics of

272 the subjects are shown in Table 1. None of the subjects reported pain or discomfort during NMES or PMS condition. 
Table 1. Basic characteristics of subjects in the NMES and PMS conditions.

\begin{tabular}{|c|c|c|c|c|c|c|}
\hline & $\mathrm{N}$ & $\begin{array}{c}\mathrm{RMT} \\
(\% \mathrm{MSO})\end{array}$ & $\begin{array}{c}\text { Intervention } \\
\text { intensity }\end{array}$ & $\begin{array}{l}\text { Pre-MEP } \\
(\mathrm{mV})\end{array}$ & $\begin{array}{l}\text { Post-MEP } \\
(\mathrm{mV})\end{array}$ & $\begin{array}{c}\text { Paired t-test } \\
\text { (MEP pre-post) }\end{array}$ \\
\hline $\begin{array}{c}\text { NMES } \\
\text { condition }\end{array}$ & 15 & $37.73 \pm 11.81$ & $\begin{array}{c}12.30 \pm 3.78 \\
(\mathrm{~mA})\end{array}$ & $1.11 \pm 0.32$ & $1.22 \pm 0.48$ & $\begin{array}{c}\mathrm{t}=0.994 ; \mathrm{df}=14 ; \\
p=0.674\end{array}$ \\
\hline $\begin{array}{c}\text { PMS } \\
\text { condition }\end{array}$ & 15 & $36.6 \pm 12.94$ & $\begin{array}{c}30.37 \pm 4.78 \\
(\% \mathrm{MSO})\end{array}$ & $1.12 \pm 0.29$ & $1.15 \pm 0.51$ & $\begin{array}{c}\mathrm{t}=0.266 ; \mathrm{df}=14 ; \\
p=0.794\end{array}$ \\
\hline $\begin{array}{l}\text { Paired t-test } \\
\text { (NMES- } \\
\text { PMS) }\end{array}$ & & $\begin{array}{c}\mathrm{t}=0.398 ; \mathrm{df}=14 ; \\
p=0.697\end{array}$ & & $\begin{array}{c}\mathrm{t}=0.129 ; \mathrm{df}=14 ; \\
p=0.900\end{array}$ & $\begin{array}{c}\mathrm{t}=0.574 ; \mathrm{df}=14 ; \\
p=0.575\end{array}$ & \\
\hline
\end{tabular}

279 RMT: resting motor threshold; NMES: neuromuscular electrical stimulation; PMS:

280 peripheral magnetic stimulation; MEP: motor evoked potential; MSO\%: Maximum

281 stimulator output\%.

282

283 Changes in cortical excitability

284 Mean RMT values measured at baseline were $37.73 \% \pm 11.81 \%$ of the maximum

285 stimulator output (MSO) for NMES sessions, and 36.60\% $\pm 12.94 \%$ of MSO for PMS

286 sessions $(p=0.697)$. Pearson's correlation analysis showed a strong linear relationship

287 between baseline RMT of the NMES and PMS conditions ( $p=0.008, \mathrm{r}=0.656$ ).

288 The pre and post-MEP amplitude of NMES and PMS conditions are presented in

289 Figure 3. Paired T-test showed that no significant difference was found between post-

290 MEP amplitude and pre-MEP amplitude both in NMES and PMS conditions ( $p_{\text {corrected }}$

$\left.291=0.674 ; p_{\text {corrected }}=0.794\right)$. No significant difference was also observed in MEP changes

292 between the two conditions.

293

a

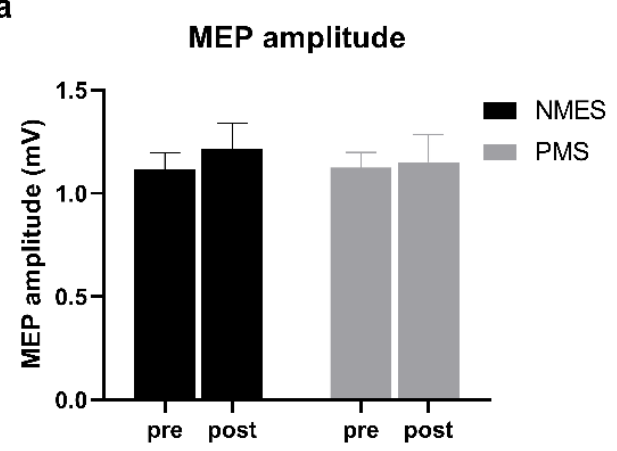

b

Correlation of MEP change between conditions

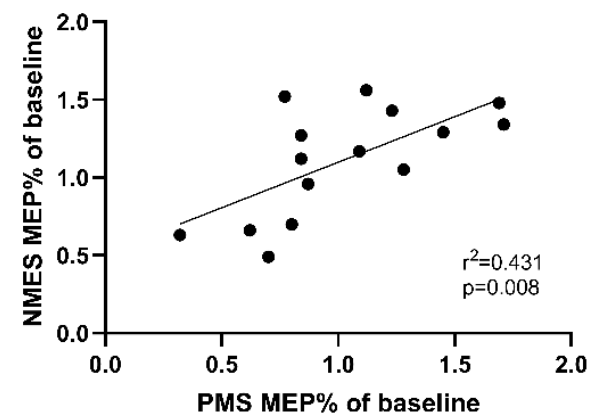

295 Figure 3. Changes in MEPs induced by NMES and PMS over the right wrist extensor

296 muscle. a MEP amplitude before and after NMES and PMS. Black bars show MEP 
amplitude by NMES. Gray bars show MEP amplitude by PMS (shown as mean with standard error). $\mathbf{b}$ Correlations of changes in MEP between NMES and PMS.

\section{fNIRS responses between different stimulation conditions}

ROI-ROI connectivity and channel-channel connectivity of resting state fNIRS were not different between the NMES and PMS conditions, the strength of functional connectivity at baseline tended to be the same between the two conditions before muscle stimulation.

In NMES condition, the $\mathrm{HbO}$ value in the PFC, right SMC and OC were decrease during stimulation period than rest period $\left(p_{\text {corrected }}<0.05\right.$ ) (figure $\left.4 a\right)$. A slight increase in the $\mathrm{HbO}$ value of ch23 and ch35 in the motor cortex of the left forearm can be observed, but there was no significant difference ( $\left.p_{\text {corrected }}>0.05\right)$. In addition, $\mathrm{HbO}$ values in ch23 and ch35 of the left forearm motor cortex increased slightly, however, no significant differences were observed. A significant decrease in the HbR value was also observed in ch4 ( $\left.p_{\text {corrected }}=0.020\right), \operatorname{ch} 15\left(p_{\text {corrected }}=0.013\right)$ of the right PFC, $\operatorname{ch} 35$ $\left(p_{\text {corrected }}=0.010\right)$ of the left SMC, and ch31 ( $\left.p_{\text {corrected }}=0.001\right)$ of the right SMC, and no HbR changes were observed in the other channels. In PMS condition, during PMS stimulation period, $\mathrm{HbO}$ values were increased in the left SMC and decreased in the OC and right SMC, however, there was not significant after Benjamini-Hochberg multiple comparisons correction ( $p_{\text {corrected }}>0.05$ ) (Figure $4 \mathrm{~b}$ ). Channel located in the SMC area during PMS stimulation period were significantly decreased than those during the rest period.

When comparing the $\mathrm{HbO}$ value and $\mathrm{HbR}$ value of each channel (total 16 channels) located in SMCs using paired T-test between the NMES and PMS stimulation periods, there was an increasing trend of the $\mathrm{HbO}$ value of the left forearm motor cortex with the PMS condition as compared with those with the NMES condition; however, the difference was not significant after multiple comparisons correction ( $\operatorname{ch} 23(p=0.005$, $\left.\left.p_{\text {corrected }}=0.083\right), \operatorname{ch} 35\left(p=0.022, p_{\text {corrected }}=0.172\right)\right)$. The HbR value under PMS condition was decreased, when compared with NMES condition, and there was no difference after correction $\left(\operatorname{ch} 23\left(p=0.007, p_{\text {corrected }}=0.116\right), \operatorname{ch} 26\left(p=0.012, p_{\text {corrected }}\right.\right.$ $\left.=0.095, \operatorname{ch} 35\left(p=0.039, p_{\text {corrected }}=0.205\right)\right)($ Figure 5 $)$.

The $\mathrm{HbO}$ maps shown in Figure 6 were plotted based on the beta values of each 329 channel, which were calculated by the general linear model during NMES and PMS 330 tasks. The $\mathrm{HbO}$ value represents the activity of the neural cortex to fNIRS responses 331 during different stimulus conditions. The hemodynamic changes of the PFC and OC showed similar trends under NMES and PMS conditions. In SMCs, the activation

333 pattern of the cortex was hemispheric, with positive activation of the left motor cortex 
334 and negative inhibition of the right motor cortex in both conditions. However, the 335 intensity of neural activation was different between NMES and PMS stimulation 336 periods.

There was no difference in ROI connection strength between NMES and PMS. Based 339 on the similarity threshold method, the brain network was constructed, and the brain 340 functional connections under different stimulation conditions were analyzed. After 341 calculating the correlation coefficient of 64 channel nodes, the similarity threshold was 342 set as $\mathrm{p} \geq 0.5,0.6,0.7$, and 0.8 , respectively. No difference was observed in the number 343 of functional connection edges between NMES and PMS under each similarity 344 threshold (Figure 7).
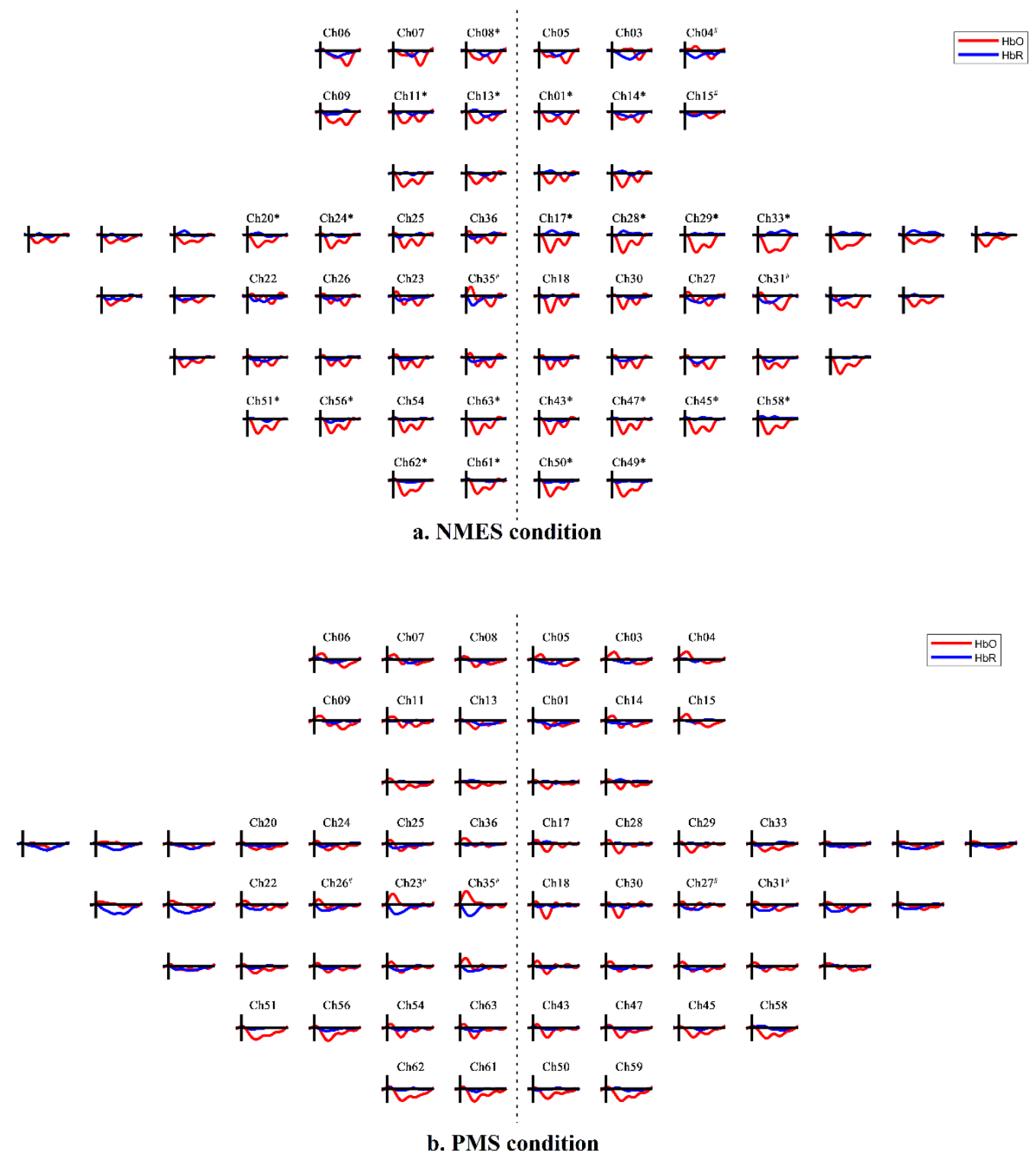
Figure 4. Averaged Hemodynamics response (0-40 s) for $\mathrm{HbO}$ (red) and $\mathrm{HbR}$ (blue) of whole channels. a during NMES condition, $\mathrm{HbO}$ in PFC, right SMC and OC regions were decreased; after multiple comparisons correction, there were significant differences in ch8 $\left(p_{\text {corrected }}=0.042\right), \operatorname{ch} 13\left(p_{\text {corrected }}=0.031\right), \operatorname{ch} 11\left(p_{\text {corrected }}=0.048\right)$ of the left PFC; $\operatorname{ch} 1\left(p_{\text {corrected }}=0.047\right)$, and ch14 $\left(p_{\text {corrected }}=0.044\right)$ of the right PFC; $\operatorname{ch} 20$ $\left(p_{\text {corrected }}=0.027\right)$, and $\operatorname{ch} 24\left(p_{\text {corrected }}=0.048\right)$ of the left SMC; $\operatorname{ch} 17\left(p_{\text {corrected }}=0.032\right)$, $\operatorname{ch} 28\left(p_{\text {corrected }}=0.032\right), \operatorname{ch} 29\left(p_{\text {corrected }}=0.049\right)$, and $\operatorname{ch} 33\left(p_{\text {corrected }}=0.044\right)$ of the right SMC; $\operatorname{ch} 51\left(p_{\text {corrected }}=0.035\right), \operatorname{ch} 56\left(p_{\text {corrected }}=0.049\right), \operatorname{ch} 61\left(p_{\text {corrected }}=0.043\right), \operatorname{ch} 62$ $\left(p_{\text {corrected }}=0.024\right)$, and ch63 $\left(p_{\text {corrected }}=0.049\right)$ of the left OC; $\operatorname{ch} 43\left(p_{\text {corrected }}=0.047\right)$, $\operatorname{ch} 45\left(p_{\text {corrected }}=0.049\right), \operatorname{ch} 47\left(p_{\text {corrected }}=0.045\right), \operatorname{ch} 49\left(p_{\text {corrected }}=0.034\right), \operatorname{ch} 50\left(p_{\text {corrected }}\right.$ $=0.028)$, and ch58 ( $p_{\text {corrected }}=0.043$ ) of the right OC. $\mathbf{b}$ during PMS condition, $\mathrm{HbO}$ increased in the left forearm motor cortex, while decreased in right SMC and OC regions. However, there was not significant after Benjamini-Hochberg multiple comparisons correction (left SMC: $\operatorname{ch} 35$ ( $\left.p=0.014, p_{\text {corrected }}=0.540\right)$; right SMC: $\operatorname{ch} 29$ $\left(p=0.038, p_{\text {corrected }}=0.303\right), \operatorname{ch} 30\left(p=0.029, p_{\text {corrected }}=0.292\right) ;$ left OC: $\operatorname{ch} 51(p=0.026$, $\left.\left.p_{\text {corrected }}=0.341\right), \operatorname{ch} 56\left(p=0.016, p_{\text {corrected }}=0.314\right)\right)$; The HbR value of $\operatorname{ch} 23\left(p_{\text {corrected }}\right.$ $=0.014), \operatorname{ch} 26\left(p_{\text {corrected }}=0.012\right), \operatorname{ch} 27\left(p_{\text {corrected }}=0.011\right), \operatorname{ch} 31\left(p_{\text {corrected }}=0.005\right)$, and $\left.\operatorname{ch} 35\left(p_{\text {corrected }}=0.005\right)\right)$. According to the MNI coordinates, channels without channel labels are not in our observation area and are not used for statistical analysis. P-values were adjusted for multiple comparisons using the Benjamini-Hochberg correction method. ${ }^{*} p_{\mathrm{HbO}}<0.05,{ }^{\#} p_{\mathrm{HbR}}<0.05$.
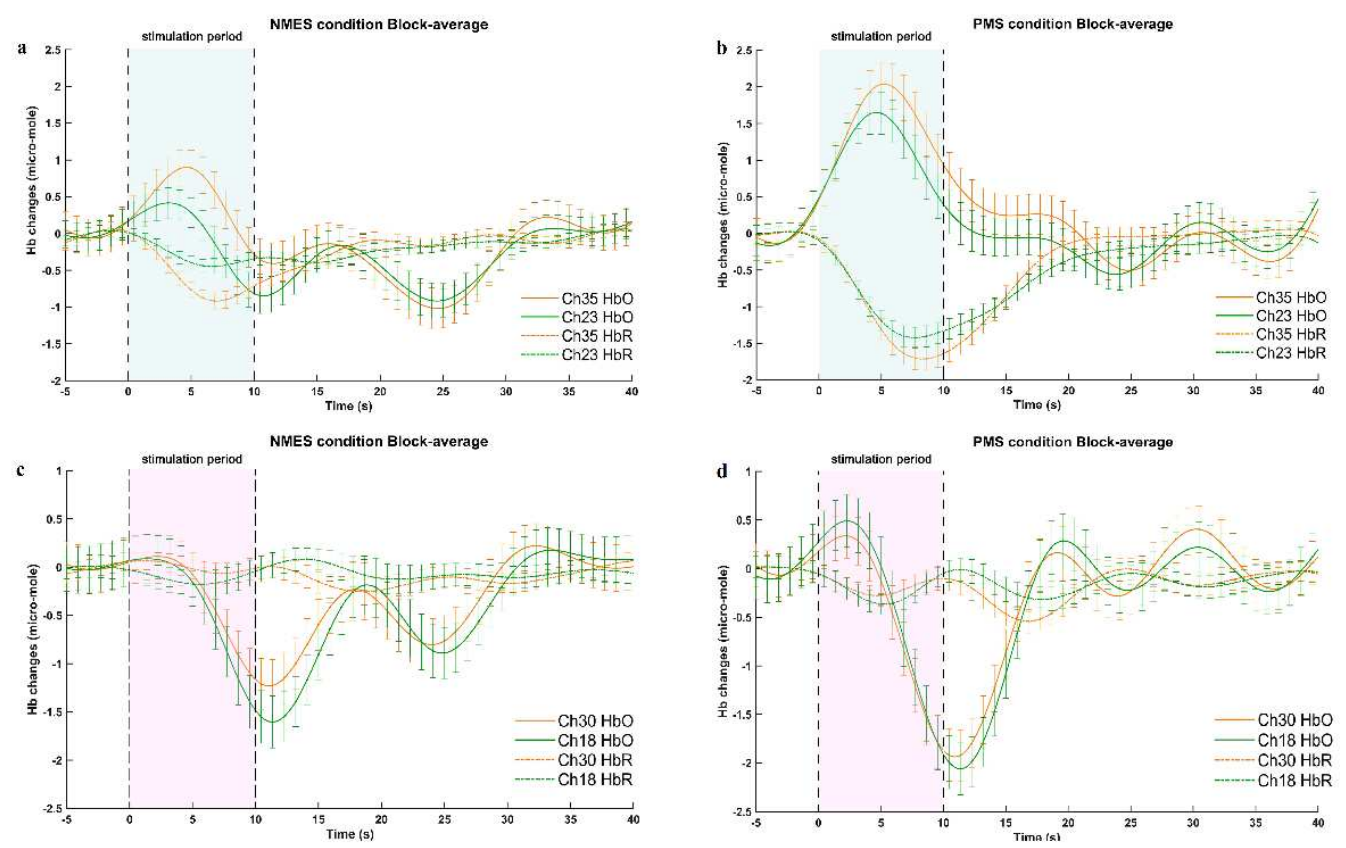
371 Figure 5. Hemoglobin time-series diagram. Comparison block-average hemodynamic response in channels 23, 35, 18, and 30 between NMES and PMS conditions. a HbO and HbR values of the left forearm motor cortex (ch23, ch25) in NMES condition. b $\mathrm{HbO}$ and $\mathrm{HbR}$ values of the left forearm motor cortex (ch23, ch25) in PMS condition. c $\mathrm{HbO}$ and $\mathrm{HbR}$ values of the right forearm motor cortex (ch18, ch30) in NMES condition. d $\mathrm{HbO}$ and $\mathrm{HbR}$ values of the right forearm motor cortex $(\operatorname{ch} 18, \operatorname{ch} 30)$ in PMS condition. The solid line represents the change in $\mathrm{HbO}$ and the dashed line represents the change in HbR. -5 to $0 \mathrm{~s}$ corresponds to the baseline period, 0 to $10 \mathrm{~s}$ corresponds to the stimulation period, 10 to $40 \mathrm{~s}$ corresponds to the rest period.
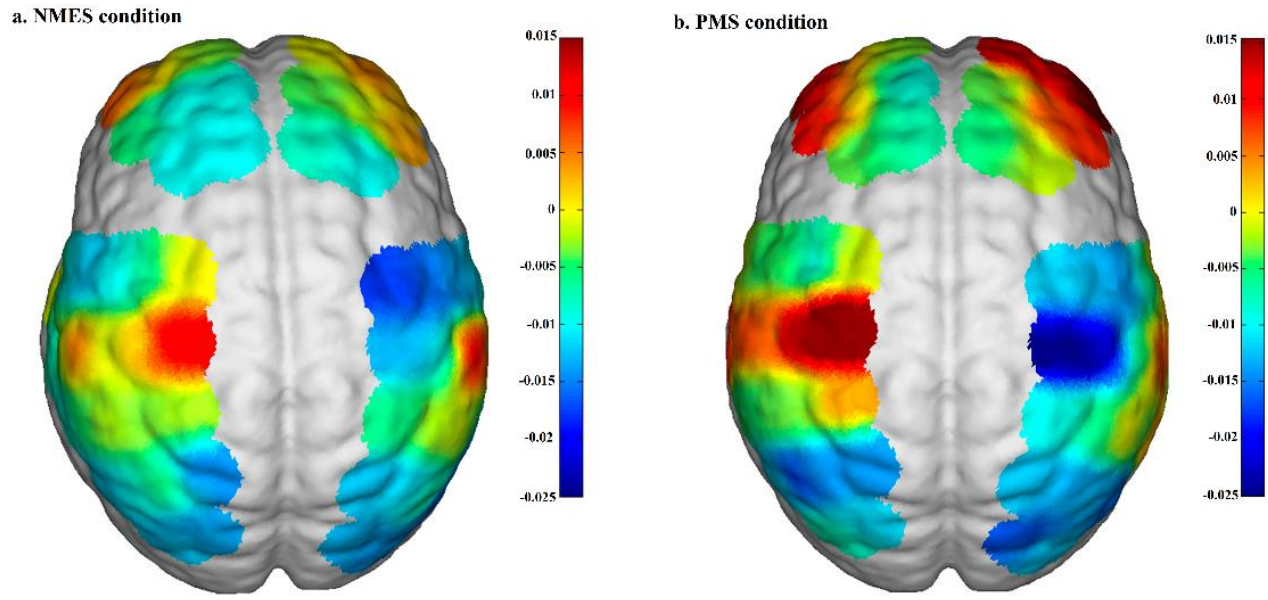

Figure 6. Cortical activation maps. HbO activation (beta scores) maps during a NMES and $\mathbf{b}$ PMS tasks. The picture comes from the group GLM analysis of the fNIRS data during stimulation task using Nirspark. 

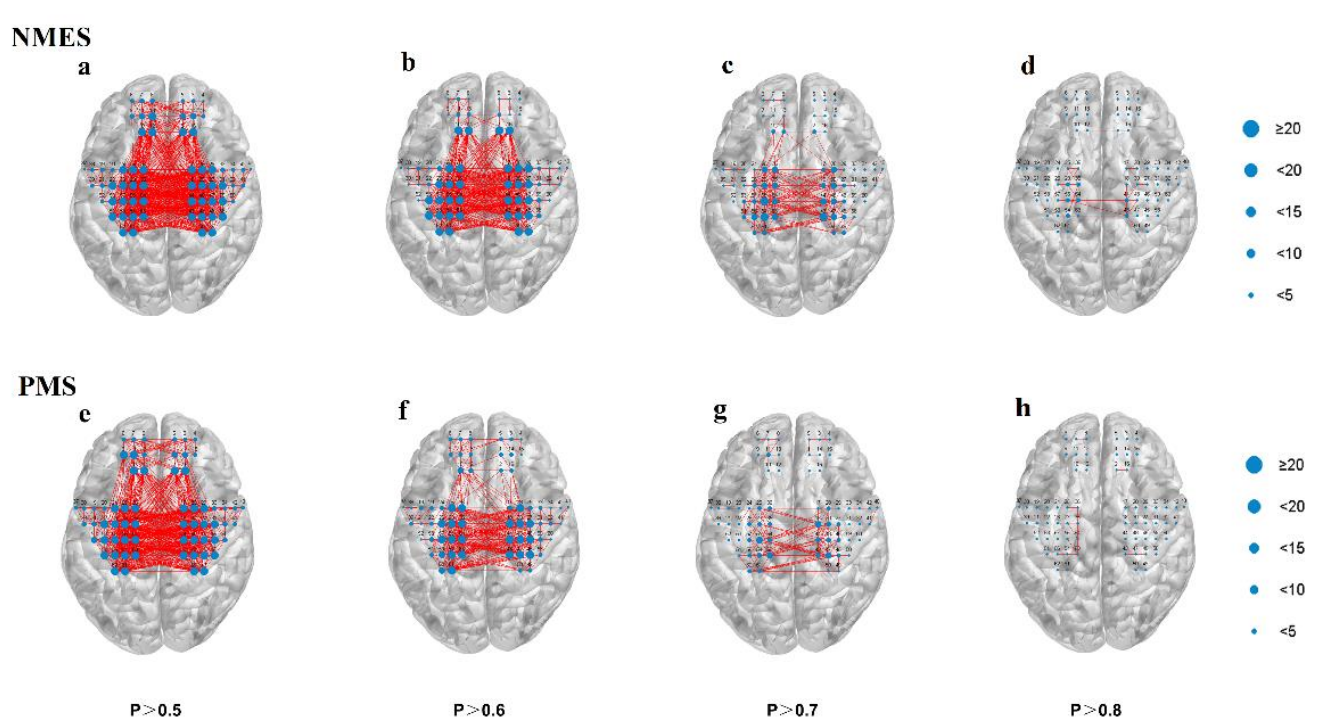

387 Figure 7. Seed-based correlation analysis. Comparison of the number of functional 388 connection edges between (a-d) NMES and (e-h) PMS conditions at thresholds of 0.5, $389 \quad 0.6,0.7$, and 0.8 .

\section{Discussion}

393 The purpose of this study was to explore the after-effects and potential mechanisms of 394 NMES and PMS on cortical activation when applied to the dominant wrist extensor 395 muscles. In the current study, cortical excitability was not changed when NMES or 396 PMS was applied to the forearm muscles to induce wrist extension, however, changes 397 in cortical activation were observed during the stimulation. NMES causes a larger area 398 of negative activation in non-stimulated brain areas, and the effect of activating the 399 corresponding cortex is weak, while PMS focuses on activating the cortex 400 corresponding to the stimulated area.

401 NMES is often applied to finger and wrist muscles to induce repetitive movements 402 to improve the efficiency of the hand in performing motor tasks by modulating the 403 cortical activity or excitability of the brain[2]. Significant cortical activation of the hand 404 sensorimotor cortex area in SMC was observed when NMES-evoked rhythmic grasp405 release hand movements by fNIRS measurements[20]. This change was unilateral, and 406 activation of the left sensorimotor network region (SMC, PMC/SMA, and S2 regions) 407 was observed when NMES-evoked right wrist extension movements[21]. However, 408 PMS as a new technique for peripheral stimulation, there is no study using fNIRS to 409 measure cortical activation patterns when PMS induces normal subjects to perform 410 hand movement tasks, let alone comparing cortical excitability (by TMS) and cortical 411 activation (by fNIRS) during NMES and PMS. Only one previous study, has used 
vibration, NMES and PMS in the ankle muscles of post-stroke patients, and used TMS to investigate the effects of different interventions on motor performance recovery and cortical excitability. The results showed that both PMS and VBI can improve ankle motion function, but only PMS can affect the excitability of M1[13]. However, the cortical activation patterns and afferent recruitment pathways of PMS and NMES, two different kinds of peripheral stimulation techniques, are still unclear.

In our study, cortical activation during PMS was more concentrated in the SMC, and the concentrations of $\mathrm{HbO}$ and $\mathrm{HbR}$ in the motor areas varied more than NMES, whereas NMES induced negative activation in more regions. The different effects of the two conditions of peripheral stimulation on cortical excitability and activation may be related to the different proprioceptive recruitment way. In contrast to NMES, PMS is thought to activate deep conduction structures and produce strong muscle contractions and a large amount of proprioception, requiring minimal skin absorption $[13,14]$. The most potential mechanism for PMS is that it induces muscle contraction by affecting muscle fibers and activating proprioceptive afferent nerves. The proprioceptive signals induced by PMS can be transmitted upward to the CNS through the full activation of mechanoreceptors (fiber groups Ia, Ib, and II) and the insufficient activation of sensorimotor nerve fibers during the rhythmic contraction and relaxation of muscles[22, 23]. Thus, the SMC can be greatly activated by the introduction of proprioceptive, then via transcallosal or subcortical interhemispheric facilitation pathway to influenced contralateral[24]. Sato et al.[25] considered that right proprioceptors stimulated by PMS flowed into the left SMC within 1s, and the influx of proprioceptive signals causes excitation of the left cerebral cortex. Cortical excitability then produces inhibitory actions in response to proprioceptive-influxinduced facilitation. The ultimate performance effect depends on the superposition of inhibition and excitation. NMES works by recruiting superficial cutaneous receptors. Electrical current via peripheral nerve transmission activates the contraction muscle fiber Ib through the depolarization of the motor axons, so that the sensory axons with lower activation threshold in the mixed nerve bundle are activated first. When the electrical stimulation intensity exceeds the MT, the muscle fiber Ia is activated. That is, sensory mediated stimulation induces excitability changes in the sensory network. Then, secondary restimulation is caused by muscle contractions[26], Furthermore, the tingling sensation will generated during NMES condition, and this meaningless sensory conduction can distract the excitability and activation of the motor cortex, and even inhibit the excitability and activation of other areas of the cortex[27]. In this study, differences were observed in the effects of PMS and NMES on cortical activation. This may be because PMS mainly activates deep proprioception, while NMES mainly recruits superficial cutaneous receptors, which produce inefficient effects and even inhibit the primary motor cortex[13, 24].

As we know, high frequency TMS increases the excitability of the ipsilateral cortex and decreases the excitability of the contralateral cortex, whereas low frequency TMS decreases the excitability of the ipsilateral cortex and increases the contralateral 
cortex[28, 29]. Numerous studies have demonstrated there is a positive correlation between cortical excitability measured by TMS and $\mathrm{HbO}$ activation measured by fNIRS. Park et al.[30] reported that during the application of 1Hz rTMS to the left M1, the concentration of $\mathrm{HbO}$ over the right $\mathrm{M} 1$ increased and the change lasted for $20 \mathrm{~min}$ after stimulation. Mochizuki et al.[31] applied inhibitory theta burst stimulation over the left S1, and the results showed a decrease in oxygenated hemoglobin and an inhibition of MEP amplitude in both the right M1 and S1. Our current study shows that both NMES and PMS applied to the wrist extensor muscles will increase the MEP amplitude of left M1. However, there was no statistical difference compared with preintervention, nor was there any significant difference in cortical excitability between conditions. Corresponding to changes in cortical excitability, during repeated passive wrist movements induced by PMS and NMES, HbO activation in the left forearm motor cortex of the SMC increased slightly but not significantly, accompanied by a significant decrease of $\mathrm{HbO}$ activation in the right.

Previous studies have demonstrated that peripheral stimulation over nerves or muscles can modulate cortical excitability $[5,32,33]$. The increase or decrease of motor cortical excitability after NMES[34] and PMS application is related to the frequency and intensity of stimulation. The intensity of NMES above the motor threshold (MT) increases cortical excitability [6, 32, 35] and decreases excitability at sensory intensities[5, 36, 37]. In particular, high-frequency of PMS facilitates motor cortical excitability[23, 25], while low frequency suppresses motor cortical excitability[25, 38]. In the present study, the intensity of NMES was above the MT, and the intensity of PMS was at a high frequency of $10 \mathrm{~Hz}$. Our study did not provide a significant regulatory effect of NMES and PMS on the cortex, which could be related to a combination of many factors, such as the anatomic site of the stimulus, the stimulus parameters, and the timing of the test $[32,39]$. Referring to previous studies, we hypothesized that the differences in the parameters used in the study may lead to a discrepancy between the results of our study and those of previous studies [40, 41]. So far, there is no consensus on the best parameters for NMES and PMS application.

A strong dose-dependent relationship exists between the intensity of NMES and cortical excitability. When NMES was applied for median nerve stimulation at $4 \mathrm{~s}$ on and $6 \mathrm{~s}$ off duty cycle and $30 \mathrm{~Hz}$ for $20 \mathrm{~min}$, the MEP amplitude could be increased with $110 \%$ MT, but no changes were found in 90\%[42]. This result is consistent with the conclusion obtained by fNIRS and fMRI[21, 43-45]. For instance, Huang et al.[45] used fNIRS to measure transient tissue oxygenation and deoxygenation changes at 10,15 , 20, 25, 30, and $35 \mathrm{~mA}$ in 43 healthy young adults during NMES. Tissue $\mathrm{HbO}$ and total hemoglobin concentrations were found to increase immediately after NMES in a dose- 
dependent manner when the current was set to $<30 \mathrm{~mA}$, a significant increase in $\mathrm{HbO}$ was observed when the current intensity was greater than $20 \mathrm{~mA}$. In our study, the intensity of electrical stimulation was relatively mild, averaging at $12.3 \mathrm{~mA}$, which was a low-intensity level compared with Huang et al.'s study, so that no significant changes in cortical excitability and cortical activation were observed. Muthalib et al.[21] observed that high current intensities (up to and slightly over the individual maximal tolerated intensity) of NMES can activate a greater area of the contralateral sensorimotor network than voluntary wrist extension movements, and balance hemispheric excitability and inhibition. In addition, the duration of NMES is an important factor affecting its effect on cortical excitability. According to a study, 20 and 40 min of NMES at $30 \mathrm{~Hz}$ intensity were strong enough to produce a "voluntary" contraction of the muscles, resulting in cortical excitability facilitation[46]. A short 10min NMES intervention in our experiment could temporarily alter $\mathrm{HbO}$ levels and activate brain regions, but it had no lasting effect. More studies have shown that $2 \mathrm{~h}$ of supra-motor threshold intensity NMES can not only increase the signal intensity of S1, M1, and PMd of the brain, but also last for 60min after the stimulation is stopped[47].

In previous PMS studies, different frequencies were used. Most studies agree that higher high-frequency can produce stronger and lasting effects than lower highfrequency. Studies revealed that PMS can effectively improve upper limb motor performance and facilitates corticospinal excitability when applied with 20 and $25 \mathrm{~Hz}$ rPMS[33, 48-50]. By contrast, there was no significant ability to alter corticospinal excitability when using lower high-frequency $(10$ or $15 \mathrm{~Hz})[25,51]$. Furthermore, studies have compared the effects of different rPMS frequencies. Gallasch et al.[12] reported that $25 \mathrm{~Hz}$ can induce more effective LTP-like plasticity in the sensorimotor cortex when compared with $10 \mathrm{~Hz}$, and no difference was found between the effect of 30 and $20 \mathrm{~Hz}$ on MEP amplitude[52]. The neuromodulation effect of PMS should not increase indefinitely with increasing frequency. There may be a level above which the effect on cortical excitability does not change, similar to NMES[45]. As we used $10 \mathrm{~Hz}$ frequency, we also observed a slight increase in MEP amplitude in both conditions, but no significant difference was observed compared with pre-intervention. Notably,

increased activation of the left motor cortex by $10 \mathrm{~Hz}$ PMS was observed with fNIRS

522 Based on the above findings, we concluded that higher than motor threshold (up to the 523 maximum tolerated current intensity), appropriate stimulation frequency, and sufficient 524 stimulation dose are needed to induce a change in cortical excitability.

\section{Limitations}


There are still some limitations in our research. First, after the intervention, fNIRS was not used to observe cerebral blood flow, and TMS was only used to assess the immediate effects of the stimulus. According to previous studies, MEP amplitude, $\mathrm{HbO}$ and HbR concentration also changed with time after stimulation. Second, magnetic stimulation equipment has the function of protecting the brain and preventing the coil from overheating that limits our choice of optimal parameters for peripheral stimulation. Also, while the parameters of NMES and PMS need to be similar, real-time fNIRS measurement is also required. The short stimulus time and insufficient intensity in our study were the main reasons for the absence of observed cortical excitability in TMS assessment. In our research, we have obtained some meaningful results, and we firmly believe that this is important to the promotion of NMES and PMS in the field of brain rehabilitation. In addition, we will perfect the experimental design to further explore the effects of NMES and PMS on the cortical activity and motor function of patients with brain injury.

\section{Conclusions}

In conclusion, this study investigated the cortical excitability and cortical activation patterns induced by different peripheral stimulation techniques. NMES and PMS applied to the right wrist extensor muscle did not modulate the cortical excitability of the M1. fNIRS detected a trend of activation in the left motor cortex during NMES and PMS stimulation period, HbO increased more with PMS compared to NMES over left SMC. Furthermore, PMS targeted to increases the activity of the contralateral SMC, while NMES increased contralateral SMC activity and negatively activated the PFC and OC.

\section{Abbreviations}

NMES: Neuromuscular electrical stimulation; NIPS: Non-invasive peripheral stimulation; MT: Motor threshold; EEG: Electroencephalography; S1: Primary sensory cortex; M1: Primary motor cortex; FDI: First dorsal interosseous; APB: Abductor pollicis brevis; PMS: Peripheral magnetic stimulation; CNS: Central nervous system; fNIRS: Functional near-infrared spectroscopy; HbO: Oxygenated hemoglobin; HbR: Deoxygenated hemoglobin; TMS: Transcranial magnetic stimulation; RMT: Resting motion threshold; MEP: Motor evoked potential; CW: Continuous-wave; PFC: Prefrontal cortex; SMC: Somatosensory motor cortex; OC: Occipital cortex; GLM: 
The authors would like to thank all the subjects for taking part in the experiment.

\section{Authors' contributions}

YFY conceived and designed the experiment, performed date pre-preprocessing and statistical data analysis and drafted the complete manuscript. WWN participated in conducting experimental data collection, recruited subject and provided data processing software support. LSJ participated in conducting experimental data collection and managed IRB approvals. LC helped edited the manuscript. TS, HRP, and WY provided experimental equipment and guided operation. ZYL conceived the idea, managed IRB approvals and helped in finalizing the manuscript. All authors read and approved the final manuscript.

\section{Funding}

This work was supported by the key projects of Shanghai Science and Technology on Biomedicine (no. 18411962300).

\section{Availability of data and materials}

The datasets generated and analyzed during the current study are available from the corresponding author on reasonable request.

\section{Declarations}

\section{Ethics approval and consent to participate}

The study design was approved by Institutional Review Board of Huashan Hospital, Fudan University (reference number: \#2019-609). All subjects gave written informed consent prior to their participation in the study.

\section{Consent for publication}

593 Not applicable

\section{Competing interests}

596 The authors declare that there are no conflicts of interest regarding the publication of 597 this paper. 


\section{References}

1. Guo XX, Fan BY, Mao YY. Effectiveness of neuromuscular electrical stimulation for wrist rehabilitation after acute ischemic stroke. Medicine (Baltimore). 2018; 97(38):e12299. https://doi.org/10.1097/MD.0000000000012299.

2. Bao SC, Khan A, Song R, Kai-Yu Tong R. Rewiring the Lesioned Brain: Electrical Stimulation for Post-Stroke Motor Restoration. J Stroke. 2020; 22(1):47-63. https://doi.org/10.5853/jos.2019.03027.

3. Mohan S, Stanbrook M, Anand A. Neuromuscular electrical stimulation to improve exercise capacity in patients with severe COPD. The Lancet Respiratory Medicine. 2016; 4(4):e14-e16. https://doi.org/10.1016/s22132600(16)00091-6.

4. Jones S, Man WD, Gao W, Higginson IJ, Wilcock A, Maddocks M. Neuromuscular electrical stimulation for muscle weakness in adults with advanced disease. Cochrane Database Syst Rev. 2016; 10(10):CD009419. https://doi.org/10.1002/14651858.CD009419.pub3.

5. Summers SJ, Schabrun SM, Marinovic W, Chipchase LS. Peripheral electrical stimulation increases corticomotor excitability and enhances the rate of visuomotor adaptation. Behav Brain Res. 2017; 322(Pt A):42-50. https://doi.org/10.1016/j.bbr.2017.01.016.

6. Schabrun SM, Ridding MC, Galea MP, Hodges PW, Chipchase LS. Primary sensory and motor cortex excitability are co-modulated in response to peripheral electrical nerve stimulation. PLoS One. 2012; 7(12):e51298. https://doi.org/10.1371/journal.pone.0051298.

7. Barker RN, Brauer SG, Barry BK, Gill TJ, Carson RG. Training-induced modifications of corticospinal reactivity in severely affected stroke survivors. Exp Brain Res. 2012; 221(2):211-221. https://doi.org/10.1007/s00221-0123163-z.

8. Liu H, Au-Yeung SSY. Corticomotor Excitability Effects of Peripheral Nerve Electrical Stimulation to the Paretic Arm in Stroke. Am J Phys Med Rehabil. 2017; 96(10):687-693. https://doi.org/10.1097/phm.0000000000000748.

9. Young W. Electrical stimulation and motor recovery. Cell Transplant. 2015; 24(3):429-446. https://doi.org/10.3727/096368915X686904.

10. Tashiro S, Mizuno K, Kawakami M, Takahashi O, Nakamura T, Suda M et al. Neuromuscular electrical stimulation-enhanced rehabilitation is associated with not only motor but also somatosensory cortical plasticity in chronic stroke patients: an interventional study. Ther Adv Chronic Dis. 2019; 10:2040622319889259. https://doi.org/10.1177/2040622319889259.

11. Beaulieu LD, Schneider C. Effects of repetitive peripheral magnetic stimulation on normal or impaired motor control. A review. Neurophysiol Clin. 2013; 43(4):251-260. https://doi.org/10.1016/j.neucli.2013.05.003. 
12. Gallasch E, Christova M, Kunz A, Rafolt D, Golaszewski S. Modulation of sensorimotor cortex by repetitive peripheral magnetic stimulation. Front Hum Neurosci. 2015; 9:407. https://doi.org/10.3389/fnhum.2015.00407.

13. Beaulieu LD, Masse-Alarie H, Camire-Bernier S, Ribot-Ciscar E, Schneider C. After-effects of peripheral neurostimulation on brain plasticity and ankle function in chronic stroke: The role of afferents recruited. Neurophysiol Clin. 2017; 47(4):275-291. https://doi.org/10.1016/j.neucli.2017.02.003.

14. Lampropoulou SI, Nowicky AV, Marston L. Magnetic versus electrical stimulation in the interpolation twitch technique of elbow flexors. Journal of sports science \& medicine. 2012; 11(4):709-718.

15. Hramov AE, Grubov V, Badarin A, Maksimenko VA, Pisarchik AN. Functional Near-Infrared Spectroscopy for the Classification of Motor-Related Brain Activity on the Sensor-Level. Sensors (Basel). 2020; 20(8). https://doi.org/10.3390/s20082362.

16. Yang M, Yang Z, Yuan T, Feng W, Wang P. A Systemic Review of Functional Near-Infrared Spectroscopy for Stroke: Current Application and Future $\begin{array}{llll}\text { Directions. Frontiers in neurology. 2019; } & \text { 10:58. }\end{array}$ https://doi.org/10.3389/fneur.2019.00058.

17. Wei Y, Chen Q, Curtin A, Tu L, Tang X, Tang Y et al. Functional near-infrared spectroscopy (fNIRS) as a tool to assist the diagnosis of major psychiatric disorders in a Chinese population. Eur Arch Psychiatry Clin Neurosci. 2020. https://doi.org/10.1007/s00406-020-01125-y.

18. Sakai K, Yasufuku Y, Kamo T, Ota E, Momosaki R. Repetitive peripheral magnetic stimulation for impairment and disability in people after stroke. Cochrane Database Syst Rev. 2019; 11(11):Cd011968. https://doi.org/10.1002/14651858.CD011968.pub3.

19. Huppert TJ, Diamond SG, Franceschini MA, Boas DA. HomER: a review of time-series analysis methods for near-infrared spectroscopy of the brain. Applied optics. 2009; 48(10):D280-298. https://doi.org/10.1364/ao.48.00d280.

20. Cortical activation change induced by neuromuscular electrical stimulation during hand movements: a functional NIRS study. 2014.

21. Muthalib M, Re R, Zucchelli L, Perrey S, Contini D, Caffini M et al. Effects of Increasing Neuromuscular Electrical Stimulation Current Intensity on Cortical Sensorimotor Network Activation: A Time Domain fNIRS Study. PLoS One. 2015; 10(7):e0131951. https://doi.org/10.1371/journal.pone.0131951.

22. Struppler A, Angerer B, Gundisch C, Havel P. Modulatory effect of repetitive peripheral magnetic stimulation on skeletal muscle tone in healthy subjects: stabilization of the elbow joint. Exp Brain Res. 2004; 157(1):59-66. https://doi.org/10.1007/s00221-003-1817-6.

23. Okudera Y, Matsunaga T, Sato M, Chida S, Hatakeyama K, Watanabe M et al. The impact of high-frequency magnetic stimulation of peripheral nerves: muscle hardness, venous blood flow, and motor function of upper extremity in 
healthy subjects. Biomedical research (Tokyo, Japan). 2015; 36(2):81-87. https://doi.org/10.2220/biomedres.36.81.

24. Struppler A, Binkofski F, Angerer B, Bernhardt M, Spiegel S, Drzezga A et al. A fronto-parietal network is mediating improvement of motor function related to repetitive peripheral magnetic stimulation: A PET-H2O15 study. Neuroimage. $\quad 2007 ; \quad 36 \quad$ Suppl 2:T174-186. https://doi.org/10.1016/j.neuroimage.2007.03.033.

25. Sato A, Liu X, Torii T, Iwahashi M, Iramina K. Modulation of motor cortex excitability by peripheral magnetic stimulation of different stimulus sites and frequencies. Conference proceedings : Annual International Conference of the IEEE Engineering in Medicine and Biology Society IEEE Engineering in Medicine and Biology Society Annual Conference. 2016; 2016:6413-6416. https://doi.org/10.1109/embc.2016.7592196.

26. Carson RG, Buick AR. Neuromuscular electrical stimulation - promoted plasticity of the human brain. The Journal of Physiology. 2019. https://doi.org/10.1113/jp278298.

27. Szecsi J, Götz S, Pöllmann W, Straube A. Force-pain relationship in functional magnetic and electrical stimulation of subjects with paresis and preserved sensation. Clin Neurophysiol. 2010; 121(9):1589-1597. https://doi.org/10.1016/j.clinph.2010.03.023.

28. Barker AT, Shields K. Transcranial Magnetic Stimulation: Basic Principles and Clinical Applications in Migraine. Headache. 2017; 57(3):517-524. https://doi.org/10.1111/head.13002.

29. Du J, Yang F, Hu J, Hu J, Xu Q, Cong $\mathrm{N}$ et al. Effects of high- and lowfrequency repetitive transcranial magnetic stimulation on motor recovery in early stroke patients: Evidence from a randomized controlled trial with clinical, neurophysiological and functional imaging assessments. Neuroimage Clin. 2019; 21:101620. https://doi.org/10.1016/j.nicl.2018.101620.

30. Park E, Kang MJ, Lee A, Chang WH, Shin YI, Kim YH. Real-time measurement of cerebral blood flow during and after repetitive transcranial magnetic stimulation: A near-infrared spectroscopy study. Neurosci Lett. 2017; 653:78-83. https://doi.org/10.1016/j.neulet.2017.05.039.

31. Mochizuki H, Furubayashi T, Hanajima R, Terao Y, Mizuno Y, Okabe S et al. Hemoglobin concentration changes in the contralateral hemisphere during and after theta burst stimulation of the human sensorimotor cortices. Exp Brain Res. 2007; 180(4):667-675. https://doi.org/10.1007/s00221-007-0884-5.

32. Mani D, Feeney DF, Enoka RM. The modulation of force steadiness by electrical nerve stimulation applied to the wrist extensors differs for young and older adults. European journal of applied physiology. 2019; 119(1):301-310. https://doi.org/10.1007/s00421-018-4025-6.

33. Asao A, Ikeda H, Nomura T, Shibuya K. Short-term session of repetitive peripheral magnetic stimulation combined with motor imagery facilitates 
corticospinal excitability in healthy human participants. Neuroreport. 2019; 30(8):562-566. https://doi.org/10.1097/wnr.0000000000001245.

34. Chipchase LS, Schabrun SM, Hodges PW. Corticospinal excitability is dependent on the parameters of peripheral electric stimulation: a preliminary study. Arch Phys Med Rehabil. 2011; 92(9):1423-1430. https://doi.org/10.1016/j.apmr.2011.01.011.

35. Howlett OA, Lannin NA, Ada L, McKinstry C. Functional electrical stimulation improves activity after stroke: a systematic review with meta-analysis. Arch Phys Med Rehabil. 2015; 96(5):934-943. https://doi.org/10.1016/j.apmr.2015.01.013.

36. Corbet T, Iturrate I, Pereira M, Perdikis S, Millan JDR. Sensory threshold neuromuscular electrical stimulation fosters motor imagery performance. Neuroimage. 2018;

176:268-276. https://doi.org/10.1016/j.neuroimage.2018.04.005.

37. Saito K, Otsuru N, Inukai Y, Kojima S, Miyaguchi S, Tsuiki S et al. Inhibitory Mechanisms in Primary Somatosensory Cortex Mediate the Effects of Peripheral Electrical Stimulation on Tactile Spatial Discrimination. Neuroscience. 2018; 384:262-274. https://doi.org/10.1016/j.neuroscience.2018.05.032.

38. Sato A, Torii T, Iwahashi M, Iramina K. Alterations in Motor Cortical Excitability Induced by Peripheral Stimulation With Magnetic Stimulation. IEEE Transactions on Magnetics. 2018; 54(11):1-4. https://doi.org/10.1109/tmag.2018.2851358.

39. Chipchase LS, Schabrun SM, Hodges PW. Peripheral electrical stimulation to induce cortical plasticity: a systematic review of stimulus parameters. Clin Neurophysiol. 2011; 122(3):456-463. https://doi.org/10.1016/j.clinph.2010.07.025.

40. Beaulieu LD, Schneider C. Repetitive peripheral magnetic stimulation to reduce pain or improve sensorimotor impairments: A literature review on parameters of application and afferents recruitment. Neurophysiol Clin. 2015; 45(3):223237. https://doi.org/10.1016/j.neucli.2015.08.002.

41. Sheffler LR, Chae J. Neuromuscular electrical stimulation in neurorehabilitation. Muscle \& nerve. 2007; 35(5):562-590. https://doi.org/10.1002/mus.20758.

42. Sasaki R, Kotan S, Nakagawa M, Miyaguchi S, Kojima S, Saito K et al. Presence and Absence of Muscle Contraction Elicited by Peripheral Nerve Electrical Stimulation Differentially Modulate Primary Motor Cortex Excitability. Front Hum Neurosci. 2017; 11:146. https://doi.org/10.3389/fnhum.2017.00146.

43. Smith GV, Alon G, Roys SR, Gullapalli RP. Functional MRI determination of a dose-response relationship to lower extremity neuromuscular electrical 
stimulation in healthy subjects. Experimental Brain Research. 2003; 150(1):3339. https://doi.org/10.1007/s00221-003-1405-9.

44. Muthalib M, Ferrari M, Quaresima V, Nosaka K. Frontal Cortex Activation During Electrical Muscle Stimulation as Revealed by Functional Near-Infrared Spectroscopy. Advances in experimental medicine and biology. 2012; 737:4549. https://doi.org/10.1007/978-1-4614-1566-4_7.

45. Huang YH, Chuang ML, Wang PZ, Chen YC, Chen CM, Sun CW. Muscle oxygenation dynamics in response to electrical stimulation as measured with near-infrared spectroscopy: A pilot study. J Biophotonics. 2019; 12(3):e201800320. https://doi.org/10.1002/jbio.201800320.

46. Andrews RK, Schabrun SM, Ridding MC, Galea MP, Hodges PW, Chipchase LS. The effect of electrical stimulation on corticospinal excitability is dependent on application duration: a same subject pre-post test design. Journal of neuroengineering and rehabilitation. 2013; 10:51. https://doi.org/10.1186/17430003-10-51.

47. Wu CW, van Gelderen P, Hanakawa T, Yaseen Z, Cohen LG. Enduring representational plasticity after somatosensory stimulation. Neuroimage. 2005; 27(4):872-884. https://doi.org/10.1016/j.neuroimage.2005.05.055.

48. Krewer C, Hartl S, Muller F, Koenig E. Effects of repetitive peripheral magnetic stimulation on upper-limb spasticity and impairment in patients with spastic hemiparesis: a randomized, double-blind, sham-controlled study. Arch Phys Med Rehabil. 2014; 95(6):1039-1047. https://doi.org/10.1016/j.apmr.2014.02.003.

49. Sollmann N, Trepte-Freisleder F, Albers L, Jung NH, Mall V, Meyer B et al. Magnetic stimulation of the upper trapezius muscles in patients with migraine A pilot study. Eur J Paediatr Neurol. 2016; 20(6):888-897. https://doi.org/10.1016/j.ejpn.2016.07.022.

50. Marz-Loose H, Siemes H. Repetitive peripheral magnetic stimulation. Treatment option for spasticity? Der Nervenarzt. 2009; 80(12):1489-1495. https://doi.org/10.1007/s00115-009-2835-9.

51. Behrens M, Mau-Möller A, Zschorlich V, Bruhn S. Repetitive Peripheral Magnetic Stimulation (15 Hz RPMS) of the Human Soleus Muscle did not Affect Spinal Excitability. Journal of sports science \& medicine. 2011; 10(1):39-44.

52. Momosaki R, Kakuda W, Yamada N, Abo M. Influence of repetitive peripheral magnetic stimulation on neural plasticity in the motor cortex related to swallowing. International Journal of Rehabilitation Research. 2016; 39(3):263266. https://doi.org/10.1097/mrr.0000000000000180. 
Figures

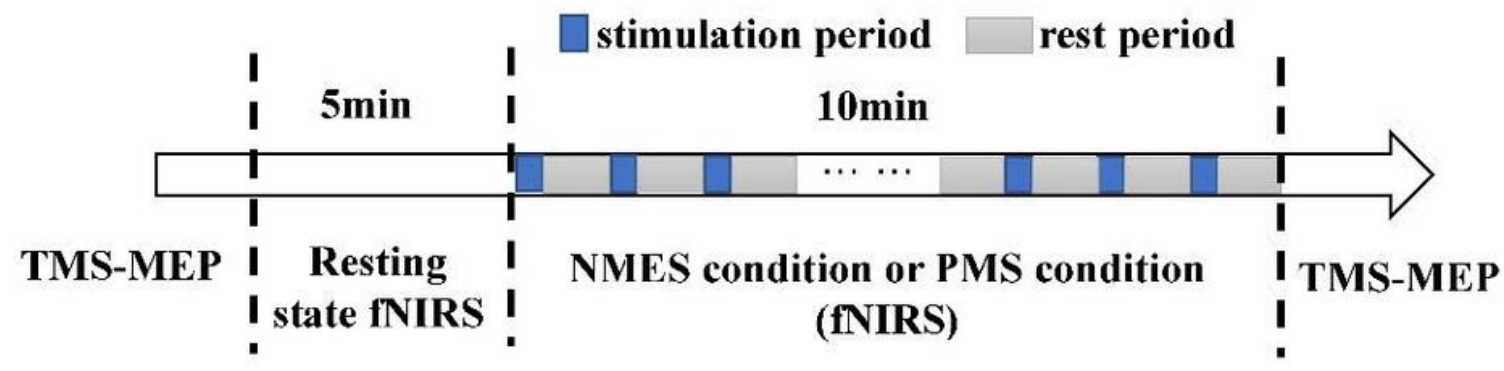

Figure 1

TMS-MEP and resting state fNIRS were assessed at the beginning of each condition, then one of the two muscle stimulation interventions (i.e. NMES or PMS) was applied, and fNIRS was also assessed during stimulation. After the intervention, cortical excitability was reassessed. 


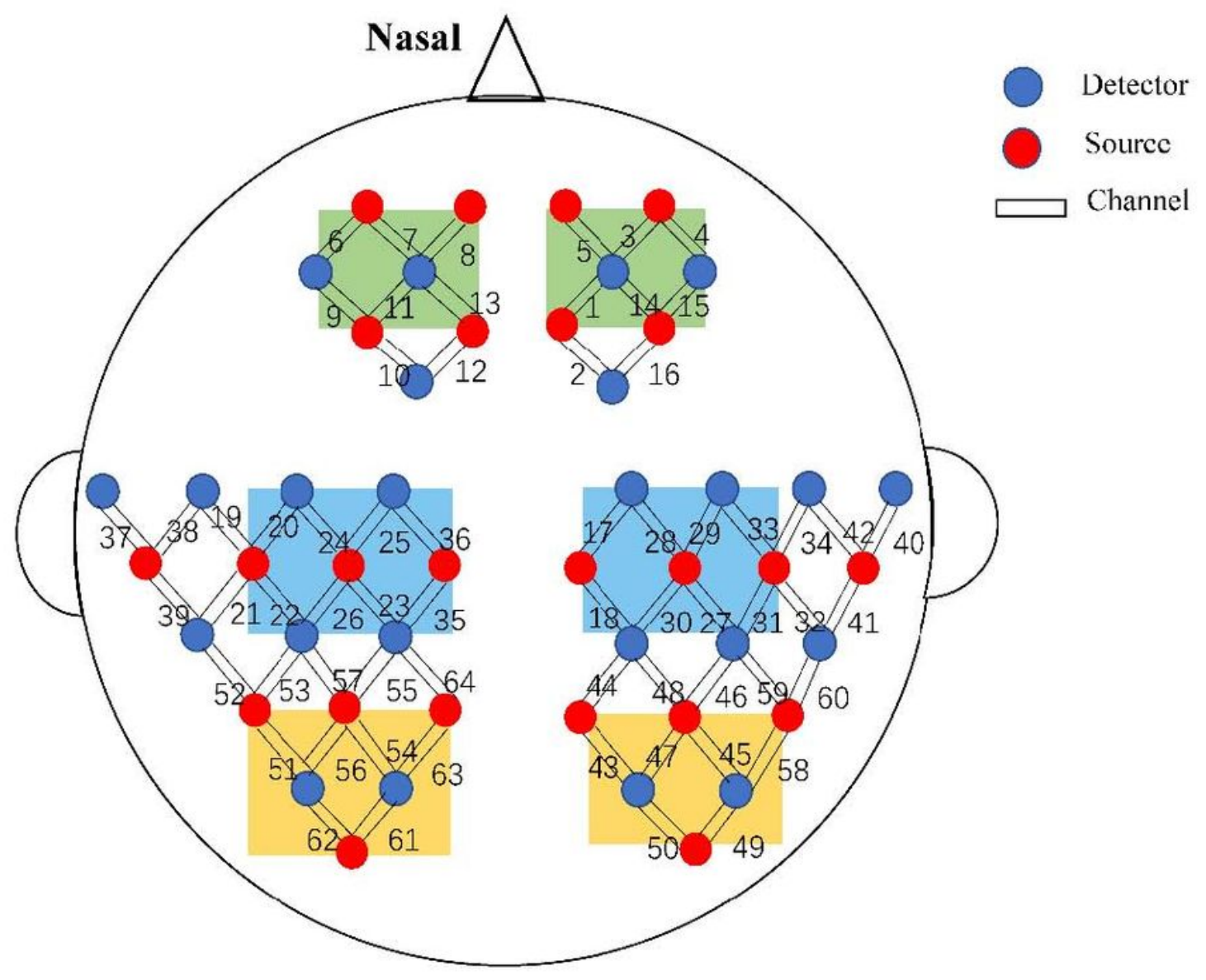

Figure 2

Probes placement. The locations of fNIRS detectors and sources are indicated by the blue and red circles, respectively, and the numbers between the circles indicate the channel numbers. The distance between the luminous source and the detector is $3 \mathrm{~cm}$. According to the MNI spatial coordinates, the channels in the green region are located in the PFC, the channels in the blue region are located in the SMC, and the channels in the yellow region are located in the OC. Channels 23 and 35 correspond to the left forearm motor cortex $\llbracket$ channels 18 and 30 correspond to the right forearm motor cortex. There are 64 channels in total, and only 40 channels in the color covered area are used for observation and analysis. 
a MEP amplitude

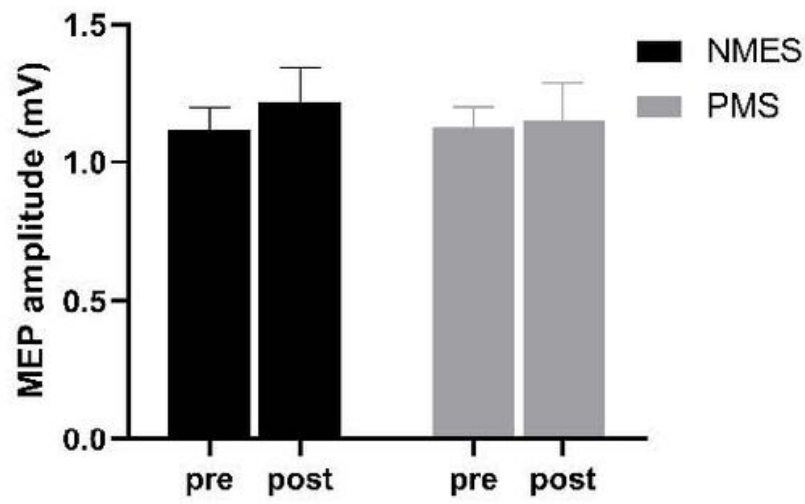

b

Correlation of MEP change between conditions

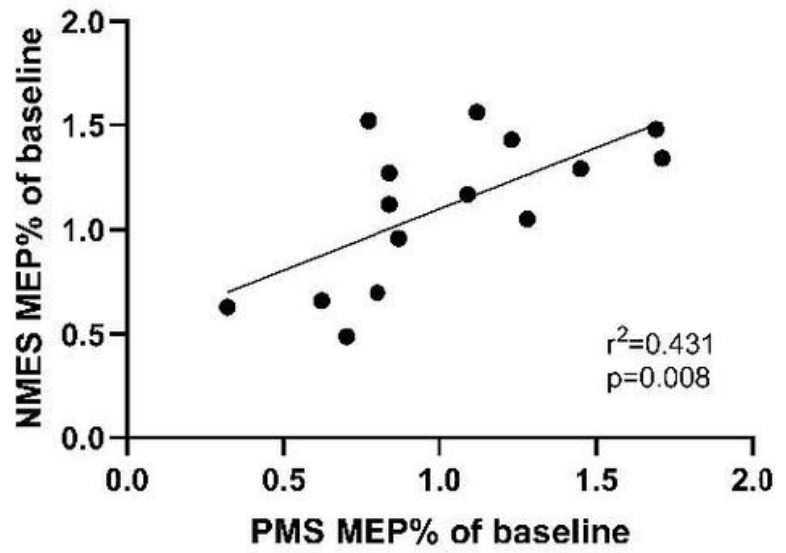

Figure 3

Changes in MEPs induced by NMES and PMS over the right wrist extensor muscle. a MEP amplitude before and after NMES and PMS. Black bars show MEP amplitude by NMES. Gray bars show MEP amplitude by PMS (shown as mean with standard error). b Correlations of changes in MEP between NMES and PMS. 

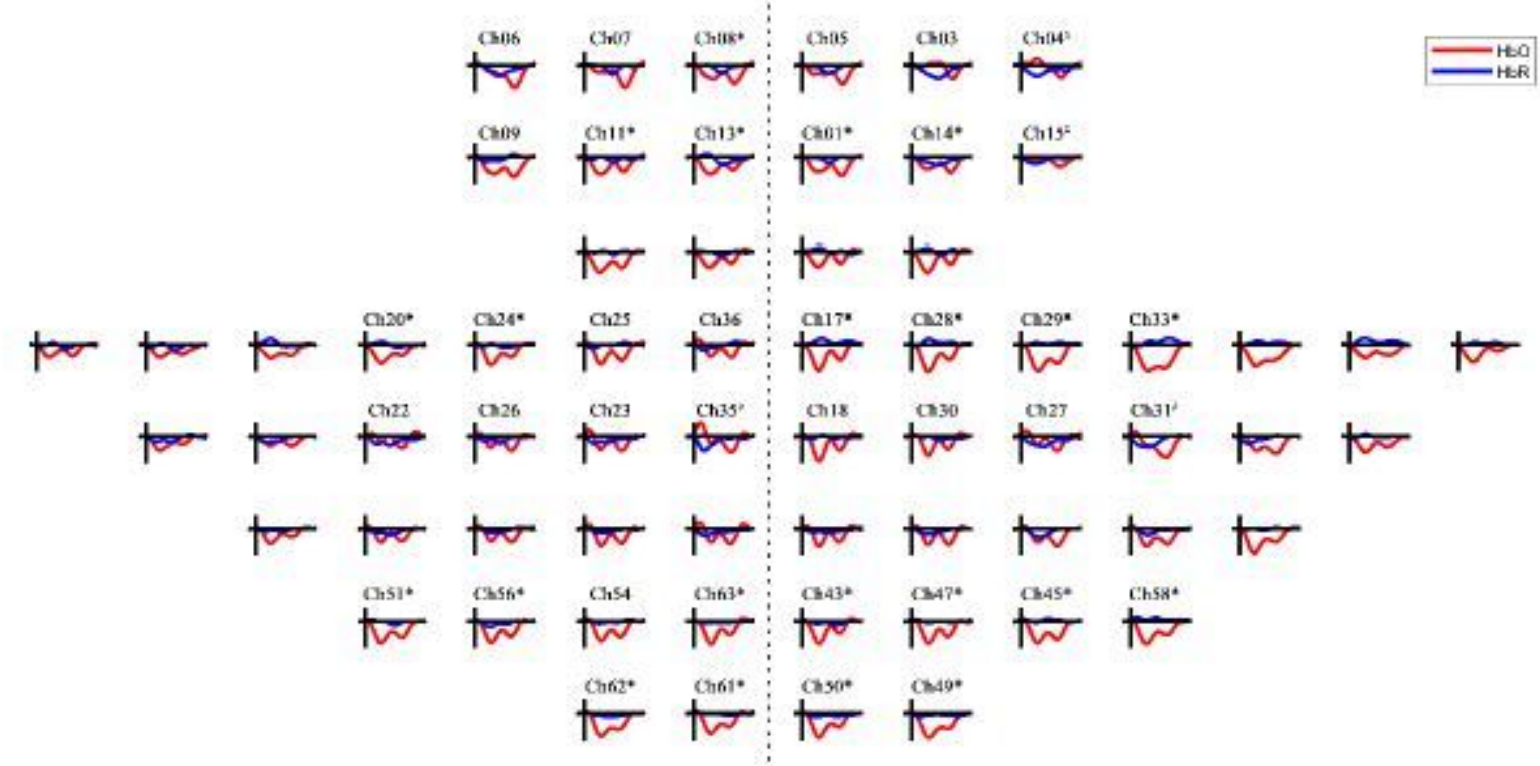

\section{a. NMES condition}

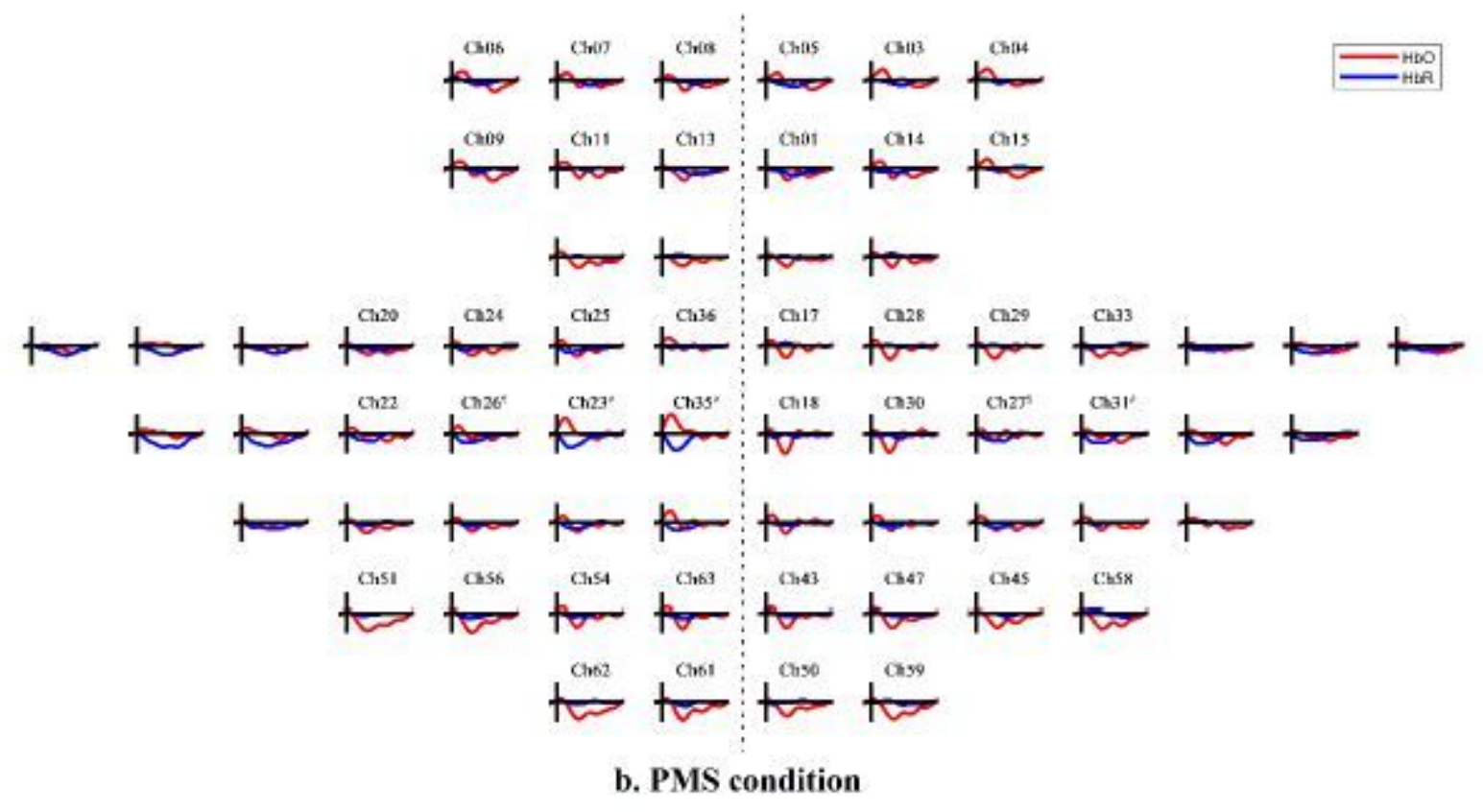

Figure 4

Averaged Hemodynamics response (0-40 s) for $\mathrm{HbO}$ (red) and $\mathrm{HbR}$ (blue) of whole channels. a during NMES condition, $\mathrm{HbO}$ in $\mathrm{PFC}$, right $\mathrm{SMC}$ and $\mathrm{OC}$ regions were decreased; after multiple comparisons correction, there were significant differences in ch8 (pcorrected $=0.042)$, ch13 (pcorrected $=0.031)$, ch11 (pcorrected $=0.048)$ of the left PFC; ch1 (pcorrected $=0.047)$, and ch14 (pcorrected $=0.044)$ of the right PFC; $\operatorname{ch} 20$ (pcorrected $=0.027)$, and ch24 (pcorrected $=0.048)$ of the left SMC; $\operatorname{ch} 17$ (pcorrected $=0.032)$, ch28 $($ pcorrected $=0.032)$, ch29 (pcorrected $=0.049)$, and ch33 (pcorrected $=0.044)$ of the right SMC; ch51 $($ pcorrected $=0.035), \operatorname{ch} 56($ pcorrected $=0.049), \operatorname{ch} 61$ (pcorrected $=0.043), \operatorname{ch} 62($ pcorrected $=$ $0.024)$, and ch63 (pcorrected $=0.049)$ of the left OC; $\operatorname{ch} 43$ (pcorrected $=0.047), \operatorname{ch} 45$ (pcorrected $=0.049)$, 
ch47 $($ pcorrected $=0.045), \operatorname{ch} 49($ pcorrected $=0.034), \operatorname{ch} 50($ pcorrected $=0.028)$, and $\operatorname{ch} 58$ (pcorrected $=$ 0.043) of the right $\mathrm{OC}$. $\mathrm{b}$ during PMS condition, $\mathrm{HbO}$ increased in the left forearm motor cortex, while decreased in right SMC and OC regions. However, there was not significant after Benjamini-Hochberg multiple comparisons correction (left SMC: $\operatorname{ch} 35(p=0.014$, pcorrected $=0.540)$; right SMC: $\operatorname{ch} 29(p=$ 0.038 , pcorrected $=0.303)$, ch30 ( $p=0.029$, pcorrected $=0.292)$; left OC: ch51 $(p=0.026$, pcorrected $=$ $0.341)$, ch56 $(p=0.016$, pcorrected $=0.314))$; The HbR value of $\operatorname{ch} 23($ pcorrected $=0.014)$, ch26 (pcorrected $=0.012), \operatorname{ch} 27$ (pcorrected $=0.011), \operatorname{ch} 31$ (pcorrected $=0.005)$, and ch35 (pcorrected $=$ 0.005)). According to the MNI coordinates, channels without channel labels are not in our observation area and are not used for statistical analysis. P-values were adjusted for multiple comparisons using the Benjamini-Hochberg correction method. ${ }^{*} \mathrm{pHbO}<0.05, \# \mathrm{pHbR}<0.05$.
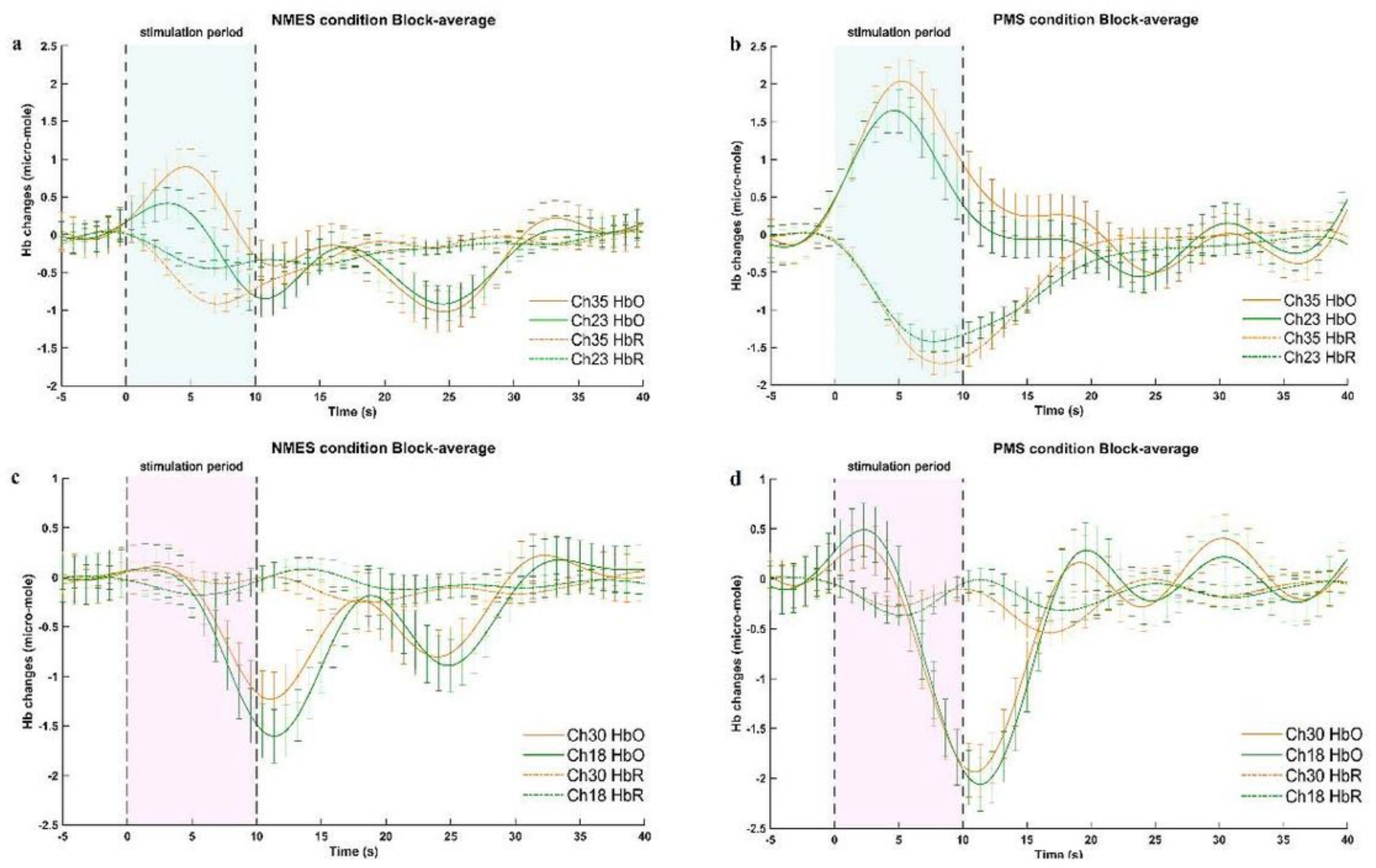

\section{Figure 5}

Hemoglobin time-series diagram. Comparison block-average hemodynamic response in channels 23,35 , 18 , and 30 between NMES and PMS conditions. a $\mathrm{HbO}$ and $\mathrm{HbR}$ values of the left forearm motor cortex (ch23, ch25) in NMES condition. b HbO and HbR values of the left forearm motor cortex (ch23, ch25) in PMS condition. $\mathrm{c} \mathrm{HbO}$ and $\mathrm{HbR}$ values of the right forearm motor cortex (ch18, ch30) in NMES condition. $\mathrm{d} \mathrm{HbO}$ and $\mathrm{HbR}$ values of the right forearm motor cortex (ch18, ch30) in PMS condition. The solid line represents the change in $\mathrm{HbO}$ and the dashed line represents the change in $\mathrm{HbR}$. -5 to 0 s corresponds to the baseline period, 0 to10 s corresponds to the stimulation period, 10 to $40 \mathrm{~s}$ corresponds to the rest period. 

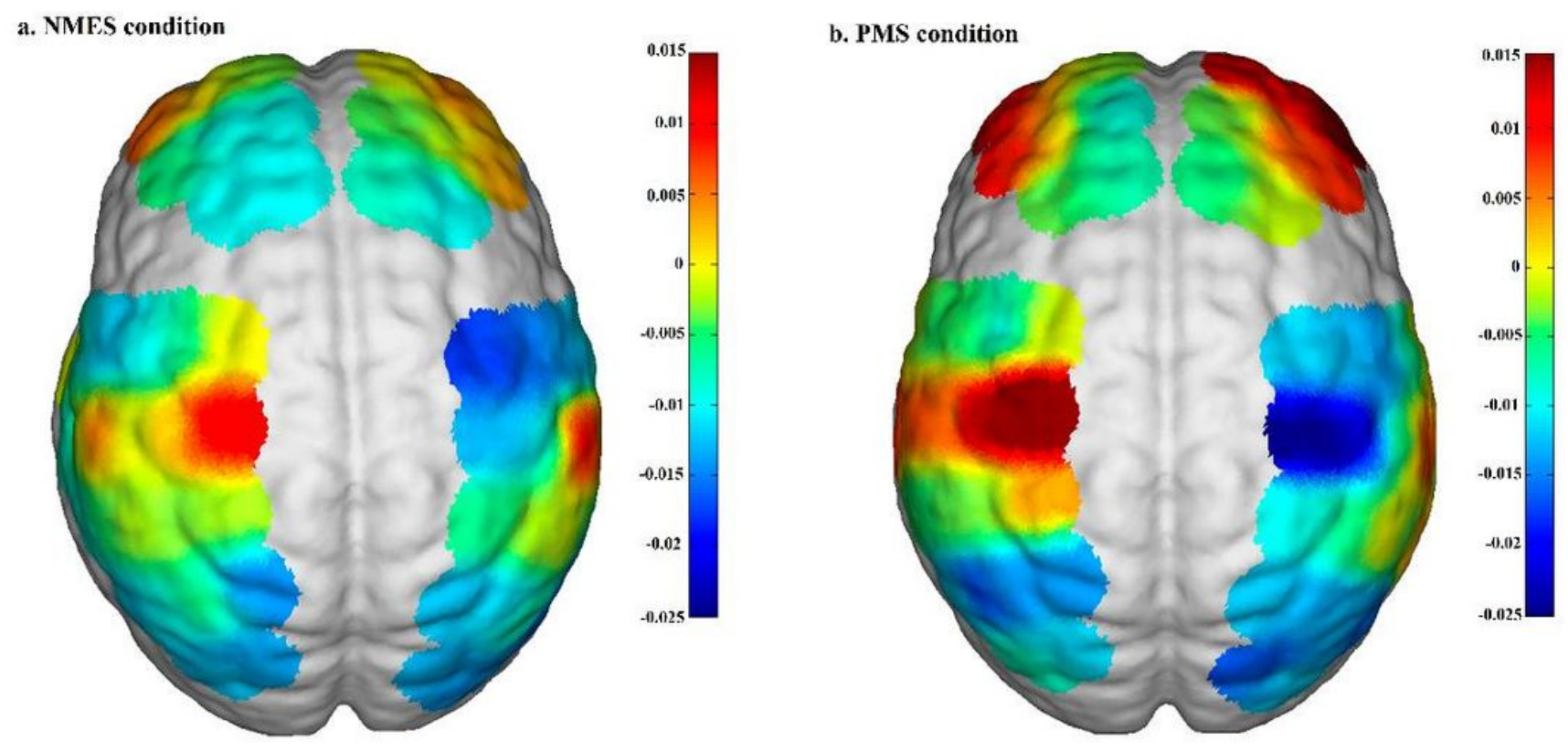

\section{Figure 6}

Cortical activation maps. HbO activation (beta scores) maps during a NMES and b PMS tasks. The picture comes from the group GLM analysis of the fNIRS data during stimulation task using Nirspark. 
NMES
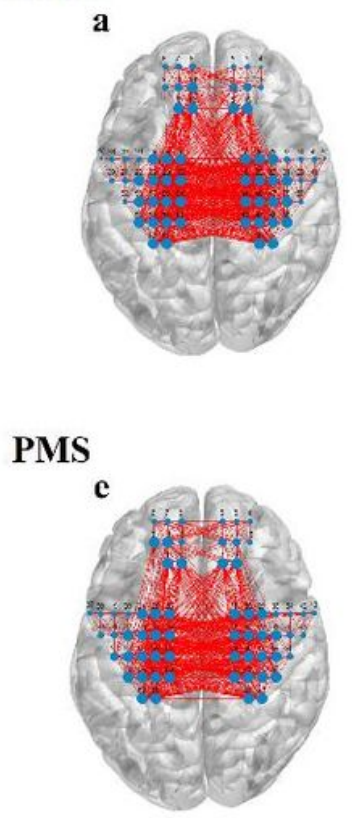

$P>0.5$
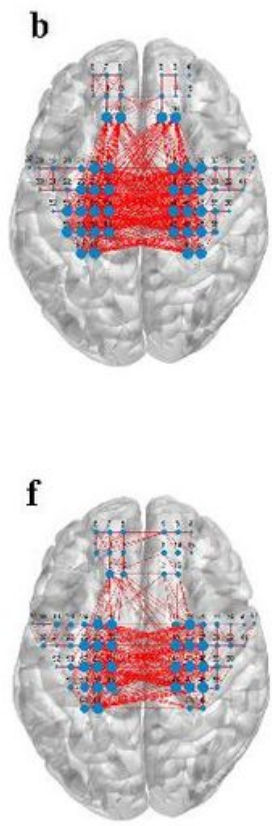

$P>0.6$

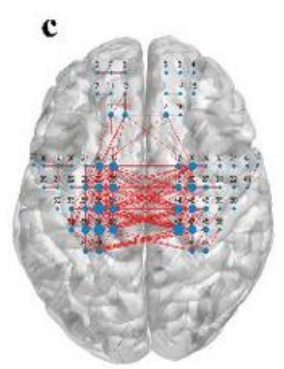

g

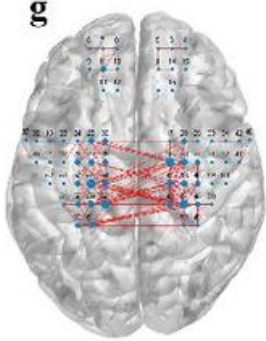

$P>0.7$ d

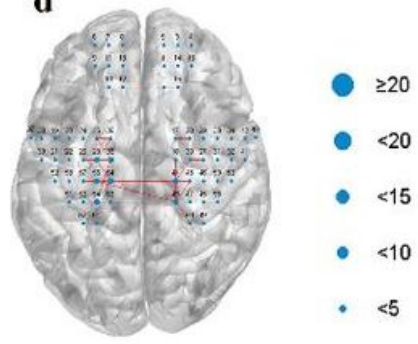

$\mathbf{h}$

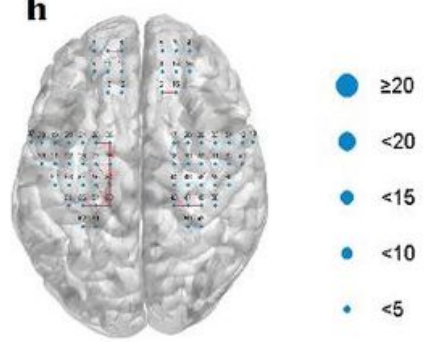

P> 0.8

Figure 7

Seed-based correlation analysis. Comparison of the number of functional connection edges between (ad) NMES and (e-h) PMS conditions at thresholds of $0.5,0.6,0.7$, and 0.8 . 